Journal of Mathematical Imaging and Vision 22: 333-353, 2005

\title{
Lattice Image Processing: A Unification of Morphological and Fuzzy Algebraic Systems
}

\author{
PETROS MARAGOS \\ National Technical University of Athens, School of Electrical \& Computer Engineering, Athens 15773, Greece \\ maragos@cs.ntua.gr
}

\begin{abstract}
This paper explores some aspects of the algebraic theory of mathematical morphology from the viewpoints of minimax algebra and translation-invariant systems and extends them to a more general algebraic structure that includes generalized Minkowski operators and lattice fuzzy image operators. This algebraic structure is based on signal spaces that combine the sup-inf lattice structure with a scalar semi-ring arithmetic that possesses generalized 'additions' and $\star$ - 'multiplications'. A unified analysis is developed for: (i) representations of translation-invariant operators compatible with these generalized algebraic structures as nonlinear sup- $\star$ convolutions, and (ii) kernel representations of increasing translation-invariant operators as suprema of erosion-like nonlinear convolutions by kernel elements. The theoretical results of this paper develop foundations for unifying large classes of nonlinear translation-invariant image and signal processing systems of the max or min type. The envisioned applications lie in the broad intersection of mathematical morphology, minimax signal algebra and fuzzy logic.
\end{abstract}

Keywords: lattices, mathematical morphology, fuzzy logic, minimax algebra

\section{Introduction}

Classic Mathematical Morphology (MM), as a field of nonlinear geometric image analysis, was developed initially by Matheron and Serra [29, 37] and their collaborators and was applied successfully to biomedical and geological problems of image analysis. In the 1980s, extensions of classic MM and connections to other fields were developed by several research groups worldwide along various directions including: applications to pattern recognition and computer vision problems; unified nonlinear filtering of the morphological/rank/stack type; multiscale image processing and shape and texture analysis; statistical analysis and optimal design of morphological filters. Detailed accounts and references can be found in books $[9,13,15,29,37$, $38]$ or tutorial papers $[11,23,24,40]$ that deal with MM. This 'classic MM' was Euclidean translation-invariant and had a max-sum arithmetic.

The need to unify its analysis tools for both binary and gray images as well as to use it for more abstract data types such as graphs led MM researchers in the late 1980 s - early 1990 s to extend its theory by generalizing the image space to a complete lattice and viewing all image transformations as lattice operators. The theoretical foundations of complete lattice MM were developed by Matheron and Serra [38] and extended further by Heijmans and Ronse $[15,16,36]$ and Roerdink [35]. A relatively new algebraic approach to morphology was developed by Keshet [18] based not on complete lattices but on inf-semilattices. The basic advance of lattice MM was to develop more general operators that shared with the standard dilation, erosion, opening and closing only a few algebraic properties. One such fundamental algebraic structure is a pair of erosion/dilation operators that form an adjunction $[15,38]$. This guarantees the formation of openings and closings via composition of the adjunction constituents. Both classic and lattice MM have focused on and exploited mainly the lattice structure (sup/inf). Although some useful operations in MM combine the sup/inf with max-plus arithmetic (e.g., Minkowski 
operations with gray structuring elements, chamfer distance transforms), such cases have always remained a minority.

In parallel to the development of MM, there has been another independent effort in the 1980's by Ritter and co-workers [32, 33], called image algebra, to unify image operations using algebraic structures such as lattices, semirings and concepts from minimax algebra. Minimax (also called 'max-plus') algebra [7] has exploited the interaction of the lattice (sup/inf) with the group structure of real addition + and has developed analogies with linear algebra that has a product-of-sums structure. There exist many applications of minimax algebra in operations research, scheduling and discrete event control systems $[6,7]$. Both image algebra and minimax algebra use concepts from lattices but have not exploited the lattice structure to the level that MM has and have not focused on the concept of lattice operators and adjunctions.

In this paper we bridge the above gaps and join MM with minimax (max-plus) algebra. Further, we generalize MM to a max- ${ }^{\star}$ signal algebra that has both a sup/inf structure and two semigroup 'multiplication'type operations $\star$ and its dual $\star$ ' that distribute over sup and inf, respectively. First we develop this generalized algebraic structure, which we call 'clodum' (complete lattice-ordered double monoid) and unifies four main cases: (1) Max-Sum MM: classic translation-invariant max-sum operations. (2) Max-Product MM: Lattice extensions of Minkowski dilations-erosions with multiplicative structuring elements. (3) Fuzzy image operations and convolutions, where $\star\left(\star^{\prime}\right)$ becomes a fuzzy intersection (union). (4) Binary translation-invariant MM: this can be seen as a special case of (1) or (3). Further, within this unified clodum structure we derive theoretical results in two areas: (A) Representations of translation-invariant operators that obey superpositions compatible with the clodum structure via generalized sup- $\star$ convolutions. (B) Representations of increasing translation-invariant operators over a clodum via generalized kernels as suprema of erosion-type inf- $\star^{\prime}$ convolutions. (C) Extensions of (A) and (B) to the special case of lattice fuzzy operators. Some parts of our results have appeared before either as special cases, e.g. the max-sum case of (A) and (B) in [21, 22], or in conference papers without proofs, e.g. the case (C) in $[25,27,28]$. Finally, we briefly discuss some benefits of our unified algebraic framework for max/min systems.

\section{Elements from Lattice and Minimax Algebra}

\subsection{Lattices}

The material in this section follows [3]. A partiallyordered set, briefly poset $(\mathcal{P}, \leq)$, is a set $\mathcal{P}$ in which a binary relation $\leq$ is defined that is a partial ordering, i.e., satisfies the following three properties for all $X, Y, Z \in \mathcal{P}$ :

(P1). $X \leq X$ (reflexive)

(P2). $X \leq Y$ and $Y \leq X$ imply $X=Y$ (antisymmetric) (P3). $X \leq Y$ and $Y \leq Z$ imply $X \leq Z$ (transitive)

If $\leq$ has the additional property that, for any two elements we have either $X \leq Y$ or $Y \leq X$, then $\mathcal{P}$ is called a totally-ordered set or chain. To every partial ordering $\leq$ on $\mathcal{P}$ there corresponds a converse partial ordering $\leq$ ' defined by ' $X \leq \leq^{\prime} Y$ iff $X \geq Y$ ”. If $(\mathcal{P}, \leq)$ is a poset, then $\left(\mathcal{P}, \leq^{\prime}\right)$ is also a poset, called the dual poset; this is the Duality Principle.

Let $\mathcal{S}$ be a subset of a poset $\mathcal{P}$. An upper bound (resp. lower bound) of $\mathcal{S}$ is an element $B \in \mathcal{P}$ such that $X \leq B$ (resp. $X \geq B$ ) for all $X \in \mathcal{S}$; if $B \in \mathcal{S}$, then it is the greatest element or maximum (resp. least element or minimum) of $\mathcal{S}$. An element $M \in \mathcal{S}$ is called maximal (resp. minimal) if there is no element in $\mathcal{S}$ that is greater (resp. smaller) than $M$. The least upper bound of $\mathcal{S}$ is called its supremum and denoted by $\sup \mathcal{S}$ or $\bigvee \mathcal{S}$. By using the duality principle, we can also define the greatest lower bound of $\mathcal{S}$, called its infimum and denoted by inf $\mathcal{S}$ or $\bigwedge \mathcal{S}$. The supremum and infimum are unique if they exist.

A lattice is a poset $(\mathcal{L}, \leq)$ any two of whose elements have a supremum, denoted by $X \vee Y$, and an infimum, denoted by $X \wedge Y$. We often denote the lattice structure by $(\mathcal{L}, \vee, \wedge)$. A lattice $\mathcal{L}$ is complete if each of its subsets has a supremum and an infimum in $\mathcal{L}$. Any nonempty complete lattice is universally bounded because it contains its greatest element (the 'unit') $I=\bigvee \mathcal{L}$ and its least element (the 'zero') $O=\bigwedge \mathcal{L}$.

Duality in Lattices: In any lattice $\mathcal{L}$, by replacing the partial ordering $\leq$ with its dual $\leq$ ' and by interchanging the roles of the supremum and infinum, i.e., by considering the dual operations $\vee^{\prime}=\wedge$ and $\wedge^{\prime}=\vee$, we can form a new lattice $\left(\mathcal{L}, \vee^{\prime}, \wedge^{\prime}\right)$, called the dual lattice and often denoted just by $\mathcal{L}^{\prime}$. The duality principle dictates that to every definition, property and statement that applies to the lattice $\mathcal{L}$ and involves its partial ordering 
Table 1. Properties of lattice operations.

\begin{tabular}{lll}
\hline Sup-semilattice & \multicolumn{1}{c}{ Inf-semilattice } & Description \\
\hline L1. $X \vee X=X$ & $\mathrm{~L}^{\prime} . X \wedge X=X$ & Idempotence \\
L2. $X \vee Y=Y \vee X$ & $\mathrm{~L} 2^{\prime} . X \wedge Y=Y \wedge X$ & Commutativity \\
L3. $X \vee(Y \vee Z)=(X \vee Y) \vee Z$ & $\mathrm{~L} 3^{\prime} . X \wedge(Y \wedge Z)=(X \wedge Y) \wedge Z$ & Associativity \\
L4. $X \vee(X \wedge Y)=X$ & $\mathrm{~L}^{\prime} . X \wedge(X \vee Y)=X$ & Absorption \\
L5. $X \leq Y \Leftrightarrow Y=X \vee Y$ & $\mathrm{~L} 5^{\prime} . X \leq Y \Leftrightarrow X=X \wedge Y$ & Consistency \\
L6. $A \wedge\left(\vee_{i \in J} X_{i}\right)=\bigvee_{i \in J}\left(A \wedge X_{i}\right)$ & $\mathrm{L} 6^{\prime} . A \vee\left(\wedge_{i \in J} X_{i}\right)=\bigwedge_{i \in J}\left(A \vee X_{i}\right)$ & Distributivity \\
& & (if $J$ is finite $\left.{ }^{\dagger}\right)$ \\
\hline
\end{tabular}

${ }^{\dagger}$ If $J$ is infinite, the lattice is called infinitely distributive.

and sup/inf, there corresponds a dual one that applies to the dual lattice $\mathcal{L}^{\prime}$ by interchanging $\leq$ with $\leq^{\prime}$ and $\vee$ with $\wedge$.

The lattice operations satisfy many properties; the four fundamentals are listed as $\left(\mathrm{L} 1, \mathrm{L1}^{\prime}\right)-\left(\mathrm{L} 4, \mathrm{~L}^{\prime}\right)$ in Table 1 . Conversely, a set $\mathcal{L}$ equipped with two binary operations $\vee$ and $\wedge$ that satisfy these first four pairs of properties is a lattice whose supremum is $\vee$, infimum is $\wedge$, and partial ordering $\leq$ is given by L5.

A lattice $\mathcal{L}$ is called distributive if property L6 of Table 1 , or equivalently its dual $\mathrm{L}^{\prime}$, holds for any finite index set $J$ and any $A, X_{i} \in \mathcal{L}$. If it also holds for an infinite index set, then the lattice is called infinitely distributive.

A lattice contains two weaker substructures, i.e., two semilattices. In general, a semilattice $(\mathcal{P}, \circ)$ is a set $\mathcal{P}$ equipped with a binary operation $\circ$ that is idempotent, commutative, and associative. Actually, any semilattice $(\mathcal{P}, \circ)$ is a poset in which the partial ordering is defined by $X \leq Y$ iff $Y=X \circ Y$ and any two elements possess a supremum ${ }^{1}$ defined by $X \vee Y=X \circ Y$; such a semilattice is called a sup-semilattice. Conversely, if $(\mathcal{P}, \leq)$ is a poset in which any two elements $X, Y$ have a supremum $X \circ Y$, then $\mathcal{P}$ is a semilattice with respect to the binary operation $\circ$. It now becomes evident that a lattice $(\mathcal{L}, \vee, \wedge)$ contains a sup-semilattice $(\mathcal{L}, \vee)$ that satisfies properties L1-L3 of Table 1 , an inf-semilattice $(\mathcal{L}, \wedge)$ that satisfies properties $\mathrm{L1}^{\prime}-\mathrm{L} 3^{\prime}$, and the two binary operations of supremum and infimum are related via properties $\mathrm{L} 4, \mathrm{~L}^{\prime}$ that make them dual to each other.

In a lattice $\mathcal{L}$ with universal bounds $O$ and $I$, an element $X \in \mathcal{L}$ is said to have a complement $X^{c} \in \mathcal{L}$ if $X \vee X^{c}=I$ and $X \wedge X^{c}=O$. If all the elements of $\mathcal{L}$ have complements, then $\mathcal{L}$ is called complemented. A lattice is called Boolean if it is complemented and distributive. In any Boolean lattice the complement of each element is unique and involutive: $\left(X^{c}\right)^{c}=X$.

A subset $\mathcal{A}$ of a complete lattice $\mathcal{L}$ is called supgenerating (resp. inf-generating) if each lattice element can be expressed as a supremum (resp. infimum) of members of $\mathcal{A}$. Such generators can be found if the lattice has atom-like elements. Specifically, an element $A \in \mathcal{L}$ is called an atom if it is a minimal element of $\mathcal{L} \backslash\{O\}$. Respectively, a dual atom is called any maximal element of $\mathcal{L} \backslash\{I\}$. Atoms may not exist in a lattice. A weaker concept is the semi-atom: a nonzero element $A \in \mathcal{L}$ is called a semi-atom if $A \leq X \vee Y$ implies $A \leq X$ or $A \leq Y$. A lattice is called atomic (resp. semiatomic) if the class of its atoms (resp. semi-atoms) is sup-generating. Dually, we can also define dual (semi) atoms, which, if they form an inf-generating class, create a dual (semi) atomic lattice.

In this paper we deal mainly with function lattices. The underlying set of these lattices is the set $\mathcal{S}=\mathbb{V}^{E}$ of all functions $F: E \rightarrow \mathbb{V}$ whose domain is an arbitrary nonempty set $E$ and range is a subset of the value set $\mathbb{V}$; this function class is also denoted by $\operatorname{Fun}(E, \mathbb{V})$. The value set $\mathbb{V}$ is always a complete lattice; let its partial order, supremum and infimum be $\leq, \vee$, and $\wedge$, respectively. Then, the complete lattice structure of $\mathbb{V}$ is also inherited by the function $\operatorname{space} \operatorname{Fun}(E, \mathbb{V})$ by extending the partial order, supremum and infimum among points in $\mathbb{V}$ to functions pointwise:

$$
\begin{aligned}
& F \leq G \Leftrightarrow F(x) \leq G(x) \quad \forall x \in E \\
&\left(\bigvee_{i \in J} F_{i}\right)(x) \triangleq \bigvee_{i \in J} F_{i}(x), \quad x \in E \\
&\left(\bigwedge_{i \in J} F_{i}\right)(x) \triangleq \bigwedge_{i \in J} F_{i}(x), \quad x \in E
\end{aligned}
$$

where $F, G, F_{i} \in \operatorname{Fun}(E, \mathbb{V})$ and $J$ is an abritrary index set. Thus the function $\operatorname{Fun}(E, \mathbb{V})$ lattice inherits 
many of the extra properties that the value lattice may have, such as its distributivity type or Boolean nature. An important example is the function lattice Fun $\left(\mathbb{R}^{m}, \overline{\mathbb{R}}\right)$, i.e. the class of $m$-dimensional extended real-valued signals.

\section{2. $\quad$ Monotone Lattice Operators}

The material in this section follows [3, 15, 38]. Let $\mathcal{L}$ be a complete distributive lattice and let $\mathcal{O}(\mathcal{L})$ be the set of all operators on $\mathcal{L}$, i.e., mappings from $\mathcal{L}$ to itself. This is an example of a function lattice where the domain and value set are both equal to $\mathcal{L}$. Given two such operators $\Psi$ and $\Phi$, we can consider their composition $\Psi \Phi(X)=\Psi(\Phi(X))$. Also we can define a partial ordering $\leq$ between them, their supremum $\Psi \vee$ $\Phi$ and infimum $\Psi \wedge \Phi$ in the obvious way induced by the corresponding operations on $\mathcal{L}$. Equipped with the above ordering, supremum and infimum, the set $\mathcal{O}(\mathcal{L})$ becomes a lattice which inherits many of the possible properties of $\mathcal{L}$, such as completeness and distributivity. Next follow some definitions for lattice operators. The identity operator is $\operatorname{id}(X)=X$. An operator $\Psi$ is called: extensive (resp. anti-extensive) if id $\leq \Psi$ (resp. $\Psi \leq$ id); idempotent if $\Psi^{2}=\Psi$ where $\Psi^{n}$ denotes the $n$-fold composition of $\Psi$ with itself; involution if $\Psi^{2}=$ id.

Of great interest are the monotone operators. A lattice operator $\Psi$ is called increasing or isotone (resp. decreasing or antitone) if it is order-preserving (resp. order-inverting), i.e., $\forall X, Y$,

increasing: $X \leq Y \Rightarrow \Psi(X) \leq \Psi(Y)$

decreasing: $X \leq Y \Rightarrow \Psi(X) \geq \Psi(Y)$

Examples of monotone operators are the morphisms. A lattice homomorphism is any operator that preserves (finite) suprema and infima; if in addition it is a bijection, then it is called an automorphism. Homomorphisms are increasing. Conversely, a dual homomorphism inverts suprema and infima. It can be shown that an operator $\Psi$ is an automorphism (resp. dual automorphism) if it is a bijection and both $\Psi$ and its inverse $\Psi^{-1}$ are increasing (resp. decreasing). Finally, a negation $v$ is a non-identity dual automorphism that is also involutive, i.e., $v^{2}=\mathrm{id}$; we often write $X^{*}=v(X)$ for the negative of a lattice element. Given a lattice operator $\Psi$, its corresponding negative operator is defined as $\Psi^{*}=\nu \Psi \nu$; i.e., $\Psi^{*}(X)=\left[\Psi\left(X^{*}\right)\right]^{*}$.

Four important types of increasing operators, which are fundamental for unifying lattice image processing, are the following:

$\delta$ is dilation iff $\delta\left(\bigvee_{i \in J} X_{i}\right)=\bigvee_{i \in J} \delta\left(X_{i}\right)$

$\varepsilon$ is erosion iff $\varepsilon\left(\bigwedge_{i \in J} X_{i}\right)=\bigwedge_{\mathrm{i} \in \mathrm{J}} \varepsilon\left(X_{i}\right)$

$\alpha$ is opening iff $\alpha$ is increasing, idempotent $\&$ anti-extensive

$\beta$ is closing iff $\beta$ is increasing, idempotent $\&$ extensive

The first two definitions require arbitrary (possibly infinite) collections $\left\{X_{i}: i \in J\right\}$ of lattice elements; hence, the dilation and erosion operators need complete lattices.

Dilations and erosions come in pairs as the following concept reveals. The operator pair $(\varepsilon, \delta)$ is called an adjunction [15, 38] if

$$
\delta(X) \leq Y \Leftrightarrow X \leq \varepsilon(Y) \quad \forall X, Y \in \mathcal{L}
$$

Given a dilation $\delta$, there is a unique erosion

$$
\varepsilon_{\delta}(Y)=\bigvee\{X: \delta(X) \leq Y\}
$$

such that $\left(\varepsilon_{\delta}, \delta\right)$ is adjunction. Conversely, given an erosion $\varepsilon$, there is a unique dilation

$$
\delta_{\varepsilon}(X)=\bigwedge\{Y: X \leq \varepsilon(Y)\}
$$

such that $\left(\varepsilon, \delta_{\varepsilon}\right)$ is adjunction. Some useful facts about adjunctions are given next:

Proposition $1([15,38])$. Let $(\varepsilon, \delta)$ be an adjunction . Then:

(i) $\delta$ is a dilation with $\delta(O)=O$ and $\varepsilon$ is an erosion with $\varepsilon(I)=I$.

(ii) $\delta \varepsilon$ is an opening, and $\varepsilon \delta$ is a closing.

(iii) If $\left(\varepsilon_{j}, \delta_{j}\right), j \in J$, are adjunctions, then $\left(\bigwedge_{j} \varepsilon_{j}\right.$, $\bigvee_{j} \delta_{j}$ ) is an adjunction.

(iv) If $\psi$ is a lattice automorphism, then $\left(\psi, \psi^{-1}\right)$ is an adjunction.

\subsection{Lattice-Ordered Monoids}

The following material follows [3, 7]. A poset, lattice, or semilattice $\mathcal{L}$ is often endowed with additional structure of the group type. Namely, $\mathcal{L}$ may have an additional binary operation, called symbolically the 'multiplication' $\star$, under which $(\mathcal{L}, \star)$ is any of the following: 
Semigroup if $\star$ is associative.

Monoid if $\star$ is associative and has identity element.

Group if $\star$ is associative, has identity, and each element has an inverse.

In addition, if $\star$ is also commutative, we obtain a commutative semigroup/monoid/group. Henceforth, we shall deal only with commutative (semi)group operations $\star$.

A lattice-ordered group is an algebra $(\mathcal{L}, \vee, \wedge, \star)$ in which $(\mathcal{L}, \vee, \wedge)$ is a lattice, $(\mathcal{L}, \star)$ is a group, and the group 'multiplication' is increasing. It follows that any group translation $X \mapsto A \star X$ is a lattice automorphism.

An algebra $(\mathcal{M}, \vee, \star)$ is called a semilattice-ordered monoid if $\mathcal{M}$ is a sup-semilattice under $\vee$, monoid under $\star$, and $\star$ distributes over $\vee$ :

$$
A \star(X \vee Y)=(A \star X) \vee(A \star Y)
$$

for all $A, B, X, Y \in \mathcal{M}$. If $\mathcal{M}$ also has an infimum $\wedge$ that (together with $\vee$ ) makes it a lattice, then $(\mathcal{M}, \vee, \wedge, \star)$ is called a lattice-ordered monoid. Suppose now that $\mathcal{M}$ is also a monoid under a 'dual multiplication' operation $\star^{\prime}$ that distributes over infimum:

$$
A \star^{\prime}(X \wedge Y)=\left(A \star^{\prime} X\right) \wedge\left(A \star^{\prime} Y\right)
$$

Now $\mathcal{M}$ has four binary operations. We call the resulting algebra $\left(\mathcal{M}, \vee, \wedge, \star, \star^{\prime}\right)$ a lattice-ordered double monoid. To the above definitions we add the word complete if $\mathcal{M}$ a complete lattice and the distributivities involved are infinite. For algebraic structures similar to the above definitions alternative names ${ }^{2}$ have been used in previous works related to discrete-event control systems.

In any lattice-ordered double monoid the distributivity of $\star$ over $\vee$ and of $\star^{\prime}$ over $\wedge$ imply that both $\star$ and $\star^{\prime}$ are increasing; i.e.,

$$
\begin{aligned}
& X \leq Y \Rightarrow A \star X \leq A \star Y \\
& X \leq Y \Rightarrow A \star^{\prime} X \leq A \star^{\prime} Y
\end{aligned}
$$

These properties imply in turn that

$$
\begin{aligned}
& A \star(X \wedge Y) \leq(A \star X) \wedge(A \star Y) \\
& A \star^{\prime}(X \vee Y) \geq\left(A \star^{\prime} X\right) \vee\left(A \star^{\prime} Y\right)
\end{aligned}
$$

If $\star=\star$ ', we have a self-dual 'multiplication'. This always happens if $(\mathcal{M}, \star)$ is a group; in this case we obtain a lattice-ordered group, and the inequalities (8) become equalities.

\section{Minimax Signal and System Representations}

\subsection{Algebraic Structures on the Scalars}

We henceforth assume that all vector elements or signals involved in the description of the systems examined herein take their values from a set $\mathbb{V}$ of scalars, which in general will be a subset of the set $\overline{\mathbb{R}}=$ $\mathbb{R} \cup\{-\infty, \infty\}$ of extended real numbers. Under the standard real number ordering $\leq, \mathbb{V}$ is a chain, and $\bigvee$ and $\bigwedge$ become the standard supremum and infimum on the reals. We assume that $\mathbb{V}$ is universally bounded, i.e., contains its least $\mathbb{V}_{\text {inf }} \triangleq \bigwedge \mathbb{V}$ and greatest element $\mathbb{V}_{\text {sup }} \triangleq \bigvee \mathbb{V}$. For the unified lattice signal processing model we need to equip $\mathbb{V}$ with four binary operations:

(A). A generalized 'addition' under which $\mathbb{V}$ becomes a complete sup-semilattice. We shall henceforth fix this 'addition' to be the standard supremum $\vee$ on $\overline{\mathbb{R}}$.

$\left(\mathrm{A}^{\prime}\right)$. A 'dual addition' which makes $\mathbb{V}$ a complete inf-semilattice and is related to the generalized 'addition' via the absorption law L4 of Table 1. The standard infimum $\wedge$ on $\overline{\mathbb{R}}$ will henceforth be this 'dual addition'.

(M). A commutative generalized 'multiplication' under which: (i) $\mathbb{V}$ is a monoid with identity element $\mathbb{V}_{\text {id }}$ and null element $\mathbb{V}_{\text {inf }}$, i.e.,

$$
a \star \mathbb{V}_{\mathrm{id}}=a, \quad a \star \mathbb{V}_{\mathrm{inf}}=\mathbb{V}_{\mathrm{inf}}, \quad \forall a \in \mathbb{V},
$$

and (ii) $\star$ is a scalar dilation, i.e., distributes over any supremum

$$
a \star\left(\bigvee_{i \in J} x_{i}\right)=\bigvee_{i \in J}\left(a \star x_{i}\right)
$$

for any (possibly infinite) index set $J$. Namely, $(\mathbb{V}, \vee, \wedge, \star)$ is a dioid [6].

$\left(\mathrm{M}^{\prime}\right)$. A commutative 'dual multiplication' $\star$ ' under which: (i) $\mathbb{V}$ is a monoid with identity $\mathbb{V}_{\text {id }}^{\prime}$ and a null element $\mathbb{V}_{\text {sup }}$, i.e.,

$$
a \star^{\prime} \mathbb{V}_{\mathrm{id}}^{\prime}=a, \quad a \star^{\prime} \mathbb{V}_{\text {sup }}=\mathbb{V}_{\text {sup }}, \quad \forall a \in \mathbb{V},
$$

and (ii) $\star^{\prime}$ is a scalar erosion, i.e., distributes over any infimum.

We group the above requirements into the following sets of conditions: 
$(\mathrm{C} 1) .(\mathbb{V}, \vee, \wedge)$ is a complete infinitely-distributive lattice.

(C2). $(\mathbb{V}, \star)$ is a commutative monoid, and $\star$ is a dilation.

(C3). $\left(\mathbb{V}, \star^{\prime}\right)$ is a commutative monoid, and $\star^{\prime}$ is an erosion.

Under the above assumptions $\left(\mathbb{V}, \vee, \wedge, \star, \star^{\prime}\right)$ becomes a commutative complete lattice-ordered double monoid, in short clodum. This will be the most general and minimally required algebraic structure we consider for the set of scalars. We avoid degenerate cases by henceforth assuming that each 'addition' is different from its corresponding 'multiplication', i.e., $\vee \neq \star$ and $\wedge \neq \star^{\prime}$. However, $\star$ may be the same as $\star^{\prime}$, in which case we have a self-dual 'multiplication'.

In some cases we may have some additional algebraic structure in $\mathbb{V}$. This occurs if we assume that $\mathbb{V}=\mathbb{V}_{\mathrm{G}} \cup\left\{\mathbb{V}_{\text {inf }}, \mathbb{V}_{\text {sup }}\right\}$ where $\left(\mathbb{V}_{\mathrm{G}}, \star\right)$ is a commutative group. Then, for each element $a \in \mathbb{V}_{\mathrm{G}}$ there exists its 'multiplicative' inverse $a^{-1}$ such that $a \star a^{-1}=\mathbb{V}_{\text {id }}$. Further, $\left(\mathbb{V}_{\mathrm{G}}, \vee, \wedge, \star, \star\right)$ is a lattice-ordered group with self-dual 'multiplication'. The 'multiplication' $\star$ and its self-dual $\star$ ' (which coincide over $\mathbb{V}_{\mathrm{G}}$ ) can be extended over the entire $\mathbb{V}$ by adding the rules in (9) and (11) involving the null elements. The resulting richer structure $(\mathbb{V}, \vee, \wedge, \star, \star)$ is called a bounded lattice-ordered group [7], in short blog.

A clodum $\mathbb{V}$ is called self-conjugate if it has a negation, i.e. an involutive dual automorphism that maps each element $a$ to its conjugate element $a^{*}$ such that

$$
\begin{aligned}
(a \vee b)^{*} & =a^{*} \wedge b^{*} \\
(a \star b)^{*} & =a^{*} \star^{\prime} b^{*}
\end{aligned}
$$

If $\mathbb{V}$ is a blog, then it becomes self-conjugate by setting

$$
a^{*}= \begin{cases}a^{-1} & \text { if } \mathbb{V}_{\text {inf }}<a<\mathbb{V}_{\text {sup }} \\ \mathbb{V}_{\text {sup }} & \text { if } a=\mathbb{V}_{\text {inf }} \\ \mathbb{V}_{\text {inf }} & \text { if } a=\mathbb{V}_{\text {sup }}\end{cases}
$$

\subsection{Signal Space and Impulse Representations}

The space $\mathcal{S}=\operatorname{Fun}(E, \mathbb{V})$ of signals with values in the lattice $\mathbb{V}$ is a special case of a function lattice. Of main importance is the case $E=\mathbb{R}^{2}$ or $E=\mathbb{Z}^{2}$, where $\mathcal{S}$ becomes the set of all image signals defined on the continuous or discrete image plane and taking scalar values in $\mathbb{V}$. But $E$ could also be a finite index set for matrix-based image processing or the set of vertices of a graph in cases of images defined on a graph. The signal space $\mathcal{S}$ becomes a complete infinitely distributive lattice if we define on it a partial ordering $\leq$, supremum $\bigvee_{i} F_{i}$, and infimum $\bigwedge_{i} F_{i}$ by extending the corresponding scalar operations to signals pointwise as in (2). If we now consider that $\mathbb{V}$ is not only a lattice but a clodum, then we can define a signal 'multiplication' $\star$ and its dual $\star^{\prime}$ pointwise

$$
\begin{gathered}
(F \star G)(x) \triangleq F(x) \star G(x), \\
\left(F \star^{\prime} G\right)(x) \triangleq F(x) \star^{\prime} G(x)
\end{gathered}
$$

Now the signal space $\mathcal{S}=\operatorname{Fun}(E, \mathbb{V})$ equipped under the four binary operations $\vee, \wedge, \star$ and $\star^{\prime}$ becomes a clodum. The corresponding signal 'additions' are provided by the supremum and infimum. Henceforth, our signal space will be the above clodum.

Viewed as a lattice, the signal clodum $\mathcal{S}$ possesses semi-atoms $q_{y, v}$ and dual semi-atoms $q_{y, v}^{\prime}$ which are the following elementary pulse signals

$$
\begin{aligned}
& q_{y, v}(x) \triangleq \begin{cases}v, & x=y \\
\mathbb{V}_{\text {inf }}, & x \neq y\end{cases} \\
& q_{y, v}^{\prime}(x) \triangleq \begin{cases}v, & x=y \\
\mathbb{V}_{\text {sup }}, & x \neq y\end{cases}
\end{aligned}
$$

Further, since $\mathcal{S}$ also has a monoid structure, we can consider translations of signal values via their ' $\star$-multiplication' by constants $v$, denoted as $\lambda_{v}$ : $a \mapsto a \star v$; we call them vertical translations, in short $V$-translations, since geometrically they affect the signal graph in the vertical direction. Similarly we can define dual vertical translations $\lambda_{v}^{\prime}: a \mapsto a \star^{\prime} v$. The scalar mappings $\lambda, \lambda^{\prime}$ can be extended to signals pointwise; we keep the same symbol for both scalar and signal operations:

$$
\begin{aligned}
& \lambda_{v}(F)(x) \triangleq \lambda_{v}[F(x)]=v \star F(x), \\
& \lambda_{v}^{\prime}(F)(x) \triangleq \lambda_{v}^{\prime}[F(x)]=v \star^{\prime} F(x)
\end{aligned}
$$

Now, the signal semi-atoms can be expressed as V-translations of only those whose height equals the identity. Namely, if we define

$$
q_{y}(x) \triangleq\left\{\begin{array}{ll}
\mathbb{V}_{\text {id }}, & x=y \\
\mathbb{V}_{\text {inf }}, & x \neq y
\end{array}, \quad q_{y}^{\prime}(x) \triangleq \begin{cases}\mathbb{V}_{\mathrm{id}}^{\prime}, & x=y \\
\mathbb{V}_{\text {sup }}, & x \neq y\end{cases}\right.
$$


as the impulse and dual impulse signals, respectively, then all semi-atoms can be expressed as V-translations of the impulse signals:

$$
q_{y, v}(x)=v \star q_{y}(x), \quad q_{y, v}^{\prime}(x)=v \star^{\prime} q_{y}^{\prime}(x)
$$

Hence, since $\mathcal{S}$ is a semi-atomic lattice, every signal $F(x)$ admits a representation as a supremum of V-translated impulses placed at all points of the signal domain or as infimum of dual V-translated impulses:

$$
F(x)=\bigvee_{y \in E} F(y) \star q_{y}(x)=\bigwedge_{y \in E} F(y) \star^{\prime} q_{y}^{\prime}(x)
$$

\subsection{Increasing Systems Invariant Under Vertical $\star$-Translations}

We are interested in increasing operators on the signal clodum $\mathcal{S}=\operatorname{Fun}(E, \mathbb{V})$. The previous V-translations $\lambda_{v}: F \mapsto v \star F$ of signals $F$ via 'multiplication' by constants $v$ are increasing operators. Actually they are dilations of the simplest type, which we shall often call elementary signal dilations. Their collection $\mathbb{T}_{v}=$ $\left\{\lambda_{v}: v \in \mathbb{V}_{\mathrm{G}}\right\}$ forms under composition a commutative monoid of signal dilations; i.e., $\lambda_{a} \lambda_{b}=\lambda_{a \star b}$, where

$$
\mathbb{V}_{\mathrm{G}}= \begin{cases}\mathbb{V} \backslash\left\{\mathbb{V}_{\text {inf }}, \mathbb{V}_{\text {sup }}\right\} & \text { if } \mathbb{V} \text { is blog } \\ \mathbb{V} & \text { if otherwise }\end{cases}
$$

A signal mapping $\psi$ is called $V$-translation invariant operator, in short $\mathbb{T}_{v}$-operator, if it commutes with any V-translation, i.e., $\psi \lambda_{v}=\lambda_{v} \psi$ for all $v$. All the above concepts apply as well for signal translations via dual 'multiplication'. Each dual V-translation $\lambda_{v}^{\prime}: F \mapsto$ $v \star^{\prime} F$ is an elementary signal erosion, and their collection $\mathbb{T}_{v}^{\prime}=\left\{\lambda_{v}^{\prime}: v \in \mathbb{V}_{\mathrm{G}}\right\}$ is a monoid of signal erosions. Namely, we call an operator dual $V$-translation invariant iff it commutes with any such dual V-translation. If $\mathbb{V}$ is a blog, the above two monoids $\mathbb{T}_{v}, \mathbb{T}_{v}^{\prime}$ become the same group of automorphisms on the signal lattice.

Important examples of increasing operators are the dilations and erosions. The following provides a decomposition of signal dilations and erosions on the function lattice $\mathcal{S}$ into suprema and infima of scalar dilations and erosions on $\mathbb{V}$, respectively.

Proposition 2 ([15]). Let $\mathbb{V}$ be a complete lattice and $E$ an arbitrary nonempty set. The pair $(\varepsilon, \delta)$ is an adjunction on the function lattice $\operatorname{Fun}(E, \mathbb{V})$ iff for every $x, y \in E$ there exists an adjunction $\left(e_{x, y}, d_{y, x}\right)$ on $\mathbb{V}$ such that

$$
\begin{aligned}
& \delta(F)(x)=\bigvee_{y \in E} d_{y, x}(F(y)), \\
& \varepsilon(G)(y)=\bigwedge_{x \in E} e_{x, y}(G(x))
\end{aligned}
$$

for $x, y \in E$ and $F, G \in \operatorname{Fun}(E, \mathbb{V})$.

In the signal clodum $\mathcal{S}$, if we consider the impulse signals $q_{y}(x)$ and their duals $q_{y}^{\prime}(x)$ in (15), we can enable the decomposition (18) by defining the scalar dilations to be

$$
d_{y, x}(v)=\delta\left(q_{y, v}\right)(x)=\delta\left(v \star q_{y}\right)(x), \quad x, y \in E, v \in \mathbb{V}
$$

and $e_{x, y}$ to be the adjoint erosion of $d_{y, x}$.

Dually we can define the scalar erosions $\varepsilon_{x, y}$ from the action of $\varepsilon$ on the dual impulses $q^{\prime}$, i.e.

$e_{x, y}(v)=\varepsilon\left(q_{x, v}^{\prime}\right)(y)=\varepsilon\left(v \star^{\prime} q_{x}\right)(y), \quad x, y \in E, v \in \mathbb{V}$

and then define the scalar dilations $d_{y, x}$ as adjoints of $e_{x, y}$.

An important outcome from the above discussion is that the output signals from dilation (resp. erosion) operators excited by V-translated impulses are sufficient for the supremal (resp. infimal) representation of the operators. Henceforth we assume that these operators are V-translation invariant. For dilations and erosions this invariance implies that they obey an interesting nonlinear superposition principle which has direct conceptual analogies with the linear superposition obeyed by linear operators. Specifically, it is simple to show that an operator $\delta$ is a $V$-translation invariant dilation iff

$$
\delta\left(\bigvee_{i \in J} c_{i} \star F_{i}\right)=\bigvee_{i \in J} c_{i} \star \delta\left(F_{i}\right), \quad c_{i} \in \mathbb{V}, F_{i} \in \mathcal{S}
$$

for any index set $J$. Dually, an operator $\varepsilon$ is a dual $V$-translation invariant erosion iff

$$
\varepsilon\left(\bigwedge_{i \in J} c_{i} \star^{\prime} F_{i}\right)=\bigwedge_{i \in J} c_{i} \star^{\prime} \varepsilon\left(F_{i}\right), \quad c_{i} \in \mathbb{V}, F_{i} \in \mathcal{S}
$$


Compare the two above nonlinear superpositions with the linear superposition obeyed by a linear operator $\psi$ :

$$
\psi\left(\sum_{i \in J} a_{i} \cdot F_{i}\right)=\sum_{i \in J} a_{i} \cdot \psi\left(F_{i}\right)
$$

where $J$ is a finite index set, $a_{i}$ are constants from a field (e.g. the set of reals or complex numbers) and $F_{i}$ are field-valued signals from a linear space.

If we assume that our operators are V-translation invariant, then their outputs obtain a simplified structure which is best described by defining next the operator's impulse responses. Given a dilation operator $\delta$, its impulse response function is the parametrized map $H_{y}: E \rightarrow \operatorname{Fun}(E, \mathbb{V})$ defined at each $y \in E$ as the output signal from $\delta$ when the input is the impulse $q_{y}$. Dually, for an erosion operator $\varepsilon$ we define its dual impulse response function $H^{\prime}$ via its outputs when excited by dual impulses:

$H_{y}(x) \triangleq \delta\left(q_{y}\right)(x), \quad H_{y}^{\prime}(x) \triangleq \varepsilon\left(q_{y}^{\prime}\right)(x), \quad x, y \in E$

The following gives a unified representation for all V-translation invariant dilations and erosions on a signal clodum.

Theorem 1. Consider a clodum $\left(\mathbb{V}, \vee, \wedge, \star, \star^{\prime}\right)$ of scalars and let $\mathcal{S}=\operatorname{Fun}(E, \mathbb{V})$ be the corresponding signal clodum space where $E$ is an arbitrary nonempty set. Then:

(a) An operator $\delta$ on $\mathcal{S}$ is a dilation invariant to $V$ translations, i.e. obeys (21), if and only if it can be expressed as

$$
\delta(F)(x)=\bigvee_{y \in E} F(y) \star H_{y}(x)
$$

where $H_{y}$ is its impulse response function in (24).

(b) An operator $\mathcal{E}$ on $\mathcal{S}$ is an erosion invariant to dual V-translations, i.e. obeys (22), if and only if it can be expressed as

$$
\varepsilon(F)(x)=\bigwedge_{y \in E} F(y) \star^{\prime} H_{y}^{\prime}(x)
$$

where $H_{y}^{\prime}$ is its dual impulse response function in (24).
Proof: 'Only If part': (a) Assume that $\delta$ is a V-translation invariant dilation. Then by representing the input signal $F$ via impulses as in (17) we obtain

$$
\delta(F)(x)=\bigvee_{y} \delta\left(F(y) \star q_{y}\right)(x)=\bigvee_{y} F(y) \star H_{y}(x)
$$

(b) If $\varepsilon$ is a dual V-translation invariant erosion, then by dual impulse representation of the input as in (17) we obtain

$\varepsilon(F)(x)=\bigwedge_{y} \varepsilon\left(F(y) \star^{\prime} q_{y}^{\prime}\right)(x)=\bigwedge_{y} F(y) \star^{\prime} H_{y}^{\prime}(x)$

'If part': (a) Consider an operator $\delta$ defined as in (25). By identifying the terms $F(y) \star H_{y}(x)$ as the scalar dilations $d_{y, x}(F(y))$ used in Proposition 2 we conclude that $\delta$ is a signal dilation. Further, since the group $\star$-'multiplication' distributes over suprema, $\delta$ commutes with $\star$-'multiplication' by constants. (b) Similarly, if an operator $\varepsilon$ is defined as in (26), by using the dual arguments of those used in (a), it follows that $\varepsilon$ is a dual V-translation invariant erosion.

Note that the operations in (25) and (26) are like adaptive nonlinear convolutions where a dilation (resp. erosion) system's output is obtained as supremum (resp. infimum) of various impulse response signals produced by exciting with impulses at all points and weighted by the input signal values via a group-like $\star$-'multiplication'.

\section{Translation-Invariant Lattice Image Processing Systems}

Henceforth we shall work with the signal clo$\operatorname{dum}\left(\mathcal{S}, \vee, \wedge, \star, \star^{\prime}\right)$ where the underlying set $\mathcal{S}=$ $\operatorname{Fun}(\mathbb{E}, \mathbb{V})$ consists of all signals with values from a scalar clodum $\mathbb{V} \subseteq \overline{\mathbb{R}}$ and defined on a multidimensional Euclidean domain $\mathbb{E}$ that is a subset of $\mathbb{R}^{m}$ or $\mathbb{Z}^{m}, m=1,2, \ldots$. We shall consider monoids of generalized signal translations, which include both horizontal and vertical translations, and shall prove that signal dilations (resp. erosions) invariant under such translations are equivalent to generalized supremal (resp. infimal) convolutions. Related adjunctions will also be found from pairs of such operators. These results generalize Heijman's work [15] where the vertical translations were constrained to form a scalar group and $\mathbb{T}$ was constrained to be group of automorphisms. 


\subsection{Generalized Translations}

The signal domain $\mathbb{E}$ possesses various commutative group structures which allow us to define corresponding horizontal motions that form two major types of Euclidean motions. The most obvious and practical choice is to set $\mathbb{E}=\mathbb{R}^{m}$ or $\mathbb{Z}^{m}$ and consider the horizontal translations on the image plane where each such operator $\mu_{h}(F)(x)=F(x-h)$ performs a planar shift of an input signal $F(x)$ along the vector $h$. The class $\mathbb{T}_{h t}=\left\{\mu_{h}: h \in \mathbb{E}\right\}$ of all horizontal translations under composition is a commutative group of automorphisms both on the Boolean lattice $\mathcal{P}(\mathbb{E})$ (the collection of all subsets of $\mathbb{E}$ ) as well as on the signal lattice $\mathcal{S}$; i.e. $\mu_{x} \mu_{y}=\mu_{x+y}$. A second choice, only for the case where $\mathbb{E}=\mathbb{R}^{2} \backslash\{(0,0)\}$, is to consider $p o$ lar motions $\mu_{r, \theta}$ : that transform the support of an input signal via a rotation by $\theta$ and a radial multiplication by $r$. The class $\mathbb{T}_{h r}=\left\{\mu_{r, \theta}: r>0, \theta \in[0,2 \pi)\right\}$ of all polar motions is also a commutative group of automorphisms; i.e. $\mu_{r, \theta} \mu_{\rho, \phi}=\mu_{r \rho, \theta+\phi}$. Both of these choices were studied in $[14,15]$. As a third possibility we can consider the group of all Euclidean planar motions, i.e. combined translations and rotations; this is a non-commutative group of automorphisms studied in [35]. Henceforth, we focus only on the commutative cases, i.e. the first two choices. However, for notational simplicity, we shall use only the horizontal translation group and write it simply as $\mathbb{T}_{h}$, even if our results will also include the case of polar motions. Note also that the horizontal translations apply to more general signal domains, both continuous and discrete.

In the previous section we worked with vertical translations $\lambda_{v}[F(x)]=F(x) \star v$ whose collection $\mathbb{T}_{v}$ forms a commutative monoid of signal dilations, as well as with dual vertical translations $\lambda_{v}^{\prime}[F(x)]=F(x) \star^{\prime} v$ which are erosions. The composition of these two (horizontal and vertical) types of translations yields a general translation $\tau$ and its dual $\tau^{\prime}$ :

$$
\begin{aligned}
& \tau_{h, v}(F)(x) \triangleq F(x-h) \star v, \\
& \tau_{h, v}^{\prime}(F)(x) \triangleq F(x-h) \star^{\prime} v
\end{aligned}
$$

Note that the horizontal and vertical translations commute:

$$
\tau_{h, v}=\mu_{h} \lambda_{v}=\lambda_{v} \mu_{h}, \quad \tau_{h, v}^{\prime}=\mu_{h} \lambda_{v}^{\prime}=\lambda_{v}^{\prime} \mu_{h}
$$

The collection of all such translations

$$
\mathbb{T}=\mathbb{T}_{h} \times \mathbb{T}_{v}=\left\{\tau_{h, v}: h \in \mathbb{E}, v \in \mathbb{V}_{\mathrm{G}}\right\}
$$

forms a monoid under composition:

$$
\tau_{x, a} \tau_{y, b}=\tau_{x+y, a \star b}
$$

If $\mathbb{V}$ is a blog, then $\mathbb{T}$ becomes a group of automorphisms. However, in the general case, $\mathbb{T}$ is just a commutative monoid of elementary signal dilations on $\mathcal{S}$. Dually, the collection $\mathbb{T}^{\prime}=\left\{\tau_{h, v}^{\prime}: h \in \mathbb{E}, v \in \mathbb{V}_{\mathrm{G}}\right\}$ forms a monoid of signal erosions. We call an operator $\psi$ translation-invariant, in short $\mathbb{T}$-invariant if it commutes with all translations $\tau \in \mathbb{T}$; i.e. $\psi \tau=\tau \psi$.

Consider now two elementary signals, called the impulse $q$ and the dual impulse $q^{\prime}$

$$
q(x) \triangleq\left\{\begin{array}{ll}
\mathbb{V}_{\mathrm{id}}, & x=\overrightarrow{0} \\
\mathbb{V}_{\mathrm{inf}}, & x \neq \overrightarrow{0}
\end{array}, \quad q^{\prime}(x) \triangleq \begin{cases}\mathbb{V}_{\mathrm{id}}^{\prime}, & x=\overrightarrow{0} \\
\mathbb{V}_{\text {sup }}, & x \neq \overrightarrow{0}\end{cases}\right.
$$

which are the pulse semi-atoms of $\mathcal{S}$ placed at the origin and with identity height. Then every signal can be represented as a supremum of translated impulses or as infimum of dual-translated impulses:

$$
F(x)=\bigvee_{y \in \mathbb{E}} F(y) \star q(x-y)=\bigwedge_{y \in \mathbb{E}} F(y) \star^{\prime} q^{\prime}(x-y)
$$

\subsection{Generalized Convolution Representation of $\mathbb{T}$-Invariant Dilations and Erosions}

Consider now a translation-invariant dilation on $\mathcal{S}$, i.e. an operator $\Delta$ that obeys the nonlinear superposition (21) and is horizontally tranlation-invariant, or equivalently distributes over suprema and obeys the $\mathbb{T}$-invariance. We call $\Delta$ a dilation translationinvariant (DTI) system. Let $H=\Delta(q)$ be the system's impulse response. We shall show next that, the DTI system's output $\Delta(F)$ due to an input signal $F$ equals the following nonlinear sup- $\star$ convolution $\star$ of the input with the impulse response:

$$
(F \circledast H)(x) \triangleq \bigvee_{y \in \mathbb{E}} F(y) \star H(x-y)
$$

Dually, consider a translation-invariant signal erosion, i.e. an operator $\mathcal{E}$ that distributes over infima and obeys 
the $\mathbb{T}^{\prime}$-invariance; we call $\mathcal{E}$ an erosion translationinvariant (ETI) system. Let $H^{\prime}=\mathcal{E}\left(q^{\prime}\right)$ be the system's dual impulse response. As shown next, the ETI system's output $\mathcal{E}(F)$ equals the following nonlinear inf $-\star^{\prime}$ convolution $\star^{\prime}$ of the input $F$ with the dual impulse response $H^{\prime}$ :

$$
\left(F \circledast \star^{\prime} H^{\prime}\right)(x) \triangleq \bigwedge_{y \in \mathbb{E}} F(y) \star^{\prime} H^{\prime}(x-y)
$$

Thus, DTI and ETI systems are represented by the above nonlinear convolutions, and conversely. Overall, we have the following fundamental result.

Theorem 2. Consider a clodum $\left(\mathbb{V}, \vee, \wedge, \star, \star^{\prime}\right)$ of scalars and let $\mathcal{S}=\operatorname{Fun}(\mathbb{E}, \mathbb{V})$ be the corresponding signal clodum space where $\mathbb{E}=\mathbb{R}^{m}$ or $\mathbb{Z}^{m}$, $m=1,2, \ldots$ Over this clodum, consider the monoid $\mathbb{T}$ of generalized translations defined in (29) and the monoid $\mathbb{T}^{\prime}$ of dual translations. Then:

(a) (DTI Systems): A signal operator $\Delta$ on $\mathcal{S}$ is a translation invariant dilation iff it can be represented as the sup- $\star$ convolution of the input signal with the system's impulse response $H=\Delta(q)$.

(b) (ETI Systems): A signal operator $\mathcal{E}$ on $\mathcal{S}$ is a translation invariant erosion iff it can be represented as the inf- $\star^{\prime}$ convolution of the input signal with the system's dual impulse response $H^{\prime}=\mathcal{E}\left(q^{\prime}\right)$.

Proof: (a) 'Only If part': Assume that $\Delta$ is a $\mathbb{T}$-invariant dilation. Then by applying $\Delta$ to the impulse sup-representation (32) of an input signal $F(x)$, we obtain

$$
\begin{aligned}
\Delta(F)(x) & =\bigvee_{y} \Delta[F(y) \star q(x-y)] \\
& =\bigvee_{y} F(y) \star \Delta[q(x-y)] \\
& =\bigvee_{y} F(y) \star H(x-y)=(F \circledast H)(x)
\end{aligned}
$$

Thus, $\Delta(F)=F \circledast H$. 'If part': Assume now that $\Delta(F)=F \circledast H$. Then, it is simple to show that the sup- $\star$ convolution $\star$ is $\mathbb{T}$-invariant, since it commutes with any horizontal translation as well as with any vertical $\star$-translation. Further, the sup- $\star$ convolution distributes over suprema, and hence it is a signal dilation. (b) The proof for ETI systems is the dual of that for DTI systems and follows by applying the $\mathbb{T}^{\prime}$-invariant erosion $\mathcal{E}$ to the impulse inf-representation (32) of the input signal $F$.

The above theorem has a direct conceptual analogy with Riesz's representation theorem for linear operators, which states that linear and horizontally translation-invariant (in short, LTI) operators are uniquely represented as linear (sum-product) convolutions of the input $F$ with their impulse response $H$ :

$$
\begin{aligned}
\psi \text { is LTI } \Leftrightarrow \psi(F)(x) & =(F * H)(x) \\
& =\sum_{y} F(y) H(x-y)
\end{aligned}
$$

\subsection{Generalized Convolution Adjunctions}

Let us now find the adjoint operators of the above nonlinear convolutions. A $\mathbb{T}$-invariant dilation $\Delta_{H}(F)=$ $F \circledast H$ can be represented via scalar dilations as

$$
\begin{aligned}
\Delta_{H}(F)(x) & =\bigvee_{y \in \mathbb{E}} F(y) \star H(x-y) \\
& =\bigvee_{y \in \mathbb{E}} \lambda_{H(x-y)}(F(y))
\end{aligned}
$$

where the V-translation $\lambda_{a}(v)=a \star v$ is a scalar dilation. Let $\lambda_{a}^{\leftarrow}$ be the scalar adjoint erosion of $\lambda_{a}$. By setting

$$
\lambda_{H(x-y)}(v)=d_{y, x}(v), \quad \lambda_{H(x-y)}^{\leftarrow}(w)=e_{x, y}(w)
$$

we can identify the scalar adjunction $\left(\lambda_{H(x-y)}^{\leftarrow}\right.$, $\left.\lambda_{H(x-y)}\right)$ of V-translations with the scalar adjunction $\left(e_{x, y}, d_{y, x}\right)$ of Proposition 2. Then, it follows that the adjoint signal erosion of $\Delta_{H}$ is

$$
\mathcal{E}_{H}(G)(y)=\bigwedge_{x \in \mathbb{E}} \lambda_{H(x-y)}^{\leftarrow}(G(x))
$$

If $\mathbb{V}$ is a blog, i.e. $\mathbb{V}_{\mathrm{G}}=V \backslash\left\{\mathbb{V}_{\text {inf }}, \mathbb{V}_{\text {sup }}\right\}$ is a group under $\star$-'multiplication', let $v^{*}$ denote the conjugate of each scalar $v \in \mathbb{V}$; this coincides with the group inverse if $v$ is a group element. Then, the scalar adjoint erosion can be written as $\lambda_{a}^{\leftarrow}(w)=a^{*} \star w$, and hence the adjoint signal erosion becomes

$$
\mathcal{E}_{H}(G)(y)=\bigwedge_{x \in \mathbb{E}} G(x) \star[H(x-y)]^{*}
$$


By interchanging $x$ with $y$ we can write this as

$$
\mathcal{E}_{H}(G)(x)=\bigwedge_{y \in \mathbb{E}} G(y) \star[H(y-x)]^{*}
$$

which, when compared with (36), reveals that the adjoint of a signal sup- $\star$ convolution has the computational structure of an inf- $\star$ correlation.

Similarly, if we consider a $\mathbb{T}^{\prime}$-erosion $\mathcal{E}_{H^{\prime}}(G)=$ $G \circledast^{\prime} H^{\prime}$ and decompose it into scalar erosions as

$$
\begin{aligned}
\mathcal{E}_{H^{\prime}}(G)(x) & =\bigwedge_{y \in \mathbb{E}} G(y) \star^{\prime} H^{\prime}(x-y) \\
& =\bigwedge_{y \in \mathbb{E}} \lambda_{H^{\prime}(x-y)}^{\prime}(G(y))
\end{aligned}
$$

where $\lambda_{a}^{\prime}(w)=a \star^{\prime} w$ are dual V-translations, then the adjoint signal dilation of $\mathcal{E}_{H^{\prime}}$ is

$$
\Delta_{H^{\prime}}(F)(y)=\bigvee_{x \in \mathbb{E}} \lambda_{H^{\prime}(x-y)}^{\prime \leftarrow}(F(x))
$$

where $\lambda_{a}^{\prime \leftarrow}$ is the scalar adjoint dilation of $\lambda_{a}^{\prime}$. If $\mathbb{V}$ is a blog and its self-dual multiplication $\star=\star^{\prime}$ is a group operation, each scalar $v$ possesses a conjugate $v^{*}$ which coincides with group inverses. Thus, for the group case, the scalar adjoint dilation becomes as $\lambda_{a}^{\prime \leftarrow}(v)=a^{*} \star v$. Then, the adjoint signal dilation becomes

$$
\Delta_{H^{\prime}}(F)(y)=\bigvee_{x \in \mathbb{E}} F(x) \star\left[H^{\prime}(x-y)\right]^{*}
$$

We see in both cases that while a $\mathbb{T}$-invariant dilation (or $\mathbb{T}^{\prime}$-invariant erosion) has the computational structure of a signal convolution, its corresponding adjoint has the structure of a nonlinear signal correlation. Now, are these adjoint operators translation-invariant? The next result describes the limits of such invariances.

Proposition 3. Let $(\varepsilon, \delta)$ be an adjunction on the clodum $\mathcal{S}$. Then:

(a) $\delta$ is invariant to any horizontal translation $\mu$ iff $\varepsilon$ is invariant to such translation; i.e. $\delta \mu=\mu \delta \Leftrightarrow$ $\varepsilon \mu=\mu \varepsilon$.

(b) Consider a scalar adjunction $(\lambda \leftarrow, \lambda)$ on $\mathbb{V}$ where $\lambda$ is a vertical translation and $\lambda \leftarrow$ is its adjoint. Then $\delta$ is invariant to a vertical translation $\lambda$ iff $\varepsilon$ is invariant to the adjoint translation $\lambda^{\leftarrow}$; i.e. $\delta \lambda=\lambda \delta \Leftrightarrow \varepsilon \lambda^{\leftarrow}=\lambda^{\leftarrow} \varepsilon$. (c) Consider a scalar adjunction $\left(\lambda^{\prime}, \lambda^{\prime \leftarrow}\right)$ on $\mathbb{V}$ where $\lambda^{\prime}$ is a dual vertical translation and $\lambda^{\prime \leftarrow}$ is its adjoint. Then $\varepsilon$ is invariant to a dual vertical translation $\lambda^{\prime}$ iff $\delta$ is invariant to the adjoint translation $\lambda^{\prime \leftarrow}$

(d) If $\mathbb{V}$ is a blog, $\delta$ is $\mathbb{T}$-invariant iff $\varepsilon$ is $\mathbb{T}$-invariant.

Proof: (a) This is a simple corollary of the fact that $\mu$ is an automorphism. (b) From the two adjunctions we obtain:

$$
\begin{gathered}
\lambda \delta(F) \leq G \Leftrightarrow \delta(F) \leq \lambda \leftarrow(G) \Leftrightarrow F \leq \varepsilon \lambda^{\leftarrow}(G) \\
\delta \lambda(F) \leq G \Leftrightarrow \lambda(F) \leq \varepsilon(G) \Leftrightarrow F \leq \lambda \leftarrow \varepsilon(G)
\end{gathered}
$$

Since $\delta \lambda=\lambda \delta$ and the above holds for all $F, G$, we conclude that $\varepsilon \lambda^{\leftarrow}=\lambda^{\leftarrow} \varepsilon$. (c) This is the dual of (b). (d) If $\mathbb{V}$ is a blog, then each vertical translation $\lambda$ is an automorphism and its adjoint $\lambda \leftarrow$ coincides with its inverse.

Concluding, our emphasis on working always with adjunctions $(\varepsilon, \delta)$ is justified by the following reasons: (i) If we have an adjunction, we can immediately create an opening $\alpha(F)=\delta \varepsilon(F)$ and a closing $\beta(F)=\varepsilon \delta(F)$, by simply concatenating the erosion and dilation. (ii) If a signal dilation (resp. erosion) is not invertible, then its adjoint erosion (resp. dilation) is the closest to an 'inverse operator'. (iii) Adjunctions provide us with many tools to analyze their constituent operators.

\subsection{Representation Theorems}

Matheron [29] proved a famous representation theorem stating that any set operator $\psi$ on $\mathcal{P}(\mathbb{E})$ that is translation-invariant (TI) and increasing can be represented as the union of erosions by all sets of its kernel $\operatorname{Ker}(\psi)=\{X: \overrightarrow{0} \in \psi(X)\}$ as well as an intersection of dilations by all sets of the kernel of the negative operator $\psi^{*}$, where $\overrightarrow{0}$ is the origin of $\mathbb{E}$. This representation theory was extended by Maragos [21] to both function and set operators by using a basis for the kernel. Specifically, if $\Psi$ is a TI signal operator on $\operatorname{Fun}(\mathbb{E}, \overline{\mathbb{R}})$ its kernel is defined by

$$
\operatorname{Ker}(\Psi)=\{F: \Psi(F)(\overrightarrow{0}) \geq 0\}
$$

and its basis consists of the minimal kernel functions. Thus, increasing TI signal operators are represented as supremum (infimum) of Minkowski erosions (dilations) by functions in their kernel, or minimally (if they 
are also upper-semicontinuous) by functions in their basis. Banon and Barrera [1] extended the kernel representation theory to non-monotone operators by proving that any TI set operator can be represented as a union of hit-miss operators. A subclass of Maragos' representations dealt with flat operators commuting with thresholding which admit sup-inf representations, i.e. supremum of local infima. Applications of this case led to the max-min representation of all rank operators and stack filters [21, 24]. The sup-inf representation of flat operators was extended by Guichard and Morel [12] to contrast-invariant operators. The applications of basis representations of increasing TI operators include: (i) System design as supremum (resp. infimum) of Minkowski erosions (resp. dilations). Such sup-inf operators have been applied to nonlinear filtering $[9,21$, 24]. (ii) Development of analytic tools for nonlinear operators. (iii) Approximation of nonlinear operators via a finite sup-inf combination of erosions-dilations, which are easy to implement, with many applications to image restoration and document image analysis [20]. (iv) Sup-inf operators applied to PDE-based modeling and implementation of multiscale image operators [12].

All the above representations dealt with classical morphology, based on the max-plus algebra. Generalized representations of increasing TI operators on complete lattices were developed by Serra [38] and Heijmans [14, 15]. In particular, Heijmans developed representations for increasing operators on complete lattices that are invariant to a commutative group of lattice automorphisms, e.g. translations or rotations. Roerdink [35] extended these results to noncommutative groups, e.g. combined translations and rotations.

In this section we extend the kernel representation theory for increasing $\mathbb{T}$-invariant operators $\Psi$ on the signal clodum $\mathcal{S}=\operatorname{Fun}(\mathbb{E}, \mathbb{V})$. The difficulty here is that $\mathbb{T}$ is a group of automorphisms when $\mathbb{V}$ is a blog, but otherwise it is only a monoid of dilations or erosions. We define the kernel of $\Psi$ by

$$
\operatorname{Ker}(\Psi) \triangleq\{F: \Psi(F) \geq q\}=\left\{F: \Psi(F)(\overrightarrow{0}) \geq \mathbb{V}_{\text {id }}\right\}
$$

where $\mathbb{V}_{\text {id }}$ is the identity element of the monoid $(\mathbb{V}, \star)$. We can reconstruct the operator $\Psi$ from its kernel by adding an extra condition: we henceforth assume that $\Psi$ also commutes with adjoint operators $\lambda \leftarrow$ of vertical translations $\lambda$. Thus, $\Psi$ is invariant to all combinations of horizontal translations $\mu$ and vertical transla- tions $\lambda$ as well as to adjoint vertical translations $\lambda \leftarrow$. We abbreviate this combined invariance by saying that $\Psi$ is $\mathbb{T}_{a}$-invariant. Obviously, if $\mathbb{V}$ is a blog, then all vertical translations $\lambda$ are automorphisms whose inverses are the adjoints $\lambda^{\leftarrow}$; hence, in the blog case, the $\mathbb{T}$-invariance is identical to the $\mathbb{T}_{a}$-invariance. But in the general case $\mathbb{T}_{a}$-invariance places an extra constraint on $\Psi$.

Now observe that, for any semi-atom

$$
q_{h, v}(x)=q(x-h) \star v=\mu_{h} \lambda_{v}(q),
$$

the adjunction $\left(\lambda_{v}^{\leftarrow}, \lambda_{v}\right)$ implies that

$$
\begin{aligned}
q_{h, v} & \leq \Psi(F) \Leftrightarrow \lambda_{v}(q) \leq \Psi\left(\mu_{-h} F\right) \Leftrightarrow q \\
& \leq \Psi\left(\lambda_{v}^{\leftarrow} \mu_{-h} F\right)
\end{aligned}
$$

Therefore, we can reconstruct the operator $\Psi$ from knowledge of its $\operatorname{kernel} \operatorname{Ker}(\Psi)$ as follows:

$$
\Psi(F)(x)=\sup \left\{v \in \mathbb{V}: \lambda_{v}^{\leftarrow} \mu_{-x}(F) \in \operatorname{Ker}(\Psi)\right\}
$$

The kernel has several properties outlined next.

Proposition 4. Consider $\mathbb{T}_{a}$-operators on the signal clodum.

(a) If $\Psi$ is increasing and $F \in \operatorname{Ker}(\Psi)$, then $G \in$ $\operatorname{Ker}(\Psi)$ for all $G \geq F$.

(b) If $\left\{\Psi_{i}: i \in J\right\}$ is an indexed family of operators, then

$$
\operatorname{Ker}(\Psi)=\bigcup_{i} \operatorname{Ker}\left(\Psi_{i}\right) \Rightarrow \Psi=\bigvee_{i} \Psi_{i}
$$

(c) $\operatorname{Ker}\left(\bigvee_{i} \Psi_{i}\right) \subseteq \bigcup_{i} \operatorname{Ker}\left(\Psi_{i}\right)$.

(d) $\operatorname{Ker}\left(\bigwedge_{i} \Psi_{i}\right)=\bigcap_{i} \operatorname{Ker}\left(\Psi_{i}\right)$.

(e) $\Psi_{1} \leq \Psi_{2} \Rightarrow \operatorname{Ker}\left(\Psi_{1}\right) \subseteq \operatorname{Ker}\left(\Psi_{2}\right)$.

Proof: (a) If $\Psi$ is increasing, $F \in \operatorname{Ker}(\Psi)$ and $F \leq G$, we have $q \leq \Psi(F) \leq \Psi(G)$. Therefore, $G \in \operatorname{Ker}(\Psi)$. (b) Let $\mathcal{K}=\bigcup_{i} \mathcal{K}_{i}$, where $\mathcal{K}=\operatorname{Ker}(\Psi)$ and $\mathcal{K}_{i}=$ $\operatorname{Ker}\left(\Psi_{i}\right)$. Then

$$
\begin{aligned}
\Psi(F)(x) & =\sup \left\{v: \lambda_{v}^{\leftarrow} \mu_{-x}(F) \in \mathcal{K}\right\} \\
& =\sup \left\{v: \lambda_{v}^{\leftarrow} \mu_{-x}(F) \in \mathcal{K}_{i}, \text { some } i\right\} \\
& =\sup \left\{v: \Psi_{i}\left(\lambda_{v}^{\leftarrow} \mu_{-x}(F)\right) \geq q, \text { some } i\right\} \\
& =\sup \left\{v: \lambda_{v}^{\leftarrow} \Psi_{i}\left(\mu_{-x}(F)\right) \geq q, \text { some } i\right\} \\
& =\sup \left\{v: \Psi_{i}\left(\mu_{-x}(F)\right) \geq \lambda_{v}(q), \text { some } i\right\} \\
& =\sup \left\{v: \Psi_{i}(F)(x) \geq v, \text { some } i\right\} \\
& =\bigvee_{i} \Psi_{i}(F)(x)
\end{aligned}
$$


Parts (c), (d) and (e) are straightforward corollaries of the kernel definition.

Example: Let $\Delta_{H}(F)=F \circledast H$ be a $\mathbb{T}$-invariant dilation as in (36) and let $\mathcal{E}_{H}$ be its adjoint erosion in (38). The kernel of this erosion is

$$
\begin{aligned}
\mathcal{K}_{H} \triangleq \operatorname{Ker}\left(\mathcal{E}_{H}\right) & =\left\{F: \mathcal{E}_{H}(F)(\overrightarrow{0}) \geq \mathbb{V}_{\mathrm{id}}\right\} \\
& =\left\{F: \bigwedge_{x} \lambda_{H(x)}^{\overleftarrow{\leftarrow}}(F(x)) \geq \mathbb{V}_{\mathrm{id}}\right\} \\
& =\left\{F: \lambda_{H(x)}^{\leftarrow}(F(x)) \geq \mathbb{V}_{\mathrm{id}} \forall x\right\} \\
& =\{F: \bigvee\{v: H(x) \star v \leq F(x)\} \\
& \left.\geq \mathbb{V}_{\mathrm{id}} \forall x\right\} \\
& =\{F: H(x) \leq F(x) \forall x\} \\
& =\{F: F \geq H\}
\end{aligned}
$$

It turns out that the kernel of the above simple erosion system is the building block of the kernel of a large class of increasing operators. This leads us to the following fundamental result.

Theorem 3. Let $\Psi$ be an increasing $\mathbb{T}_{a}$-invariant operator on the signal clodum $\mathcal{S}$ and let $\left(\mathcal{E}_{H}, \Delta_{H}\right)$ be adjunctions where $\Delta_{H}(F)=F \circledast H$ are sup $-\star$ convolutions by functions $H$ in the kernel of $\Psi$. Then, $\Psi$ can be represented as the supremum of all the adjoint erosions:

$$
\Psi(F)=\bigvee_{H \in \operatorname{Ker}(\Psi)} \mathcal{E}_{H}(F)
$$

Proof: Let $\mathcal{K}=\operatorname{Ker}(\Psi)$. Since $\Psi$ is increasing, if $H \in \mathcal{K}$ and $G \geq H$, then $G \in \mathcal{K}$. Thus, see (50), $\mathcal{K}_{H}=\{G: G \geq H\} \subseteq \mathcal{K}$. Hence, $\bigcup_{H \in \mathcal{K}} \mathcal{K}_{H} \subseteq \mathcal{K}$. Also, since $\{H\} \subseteq \mathcal{K}_{H}$, we have $\mathcal{K} \subseteq \bigcup_{H} \mathcal{K}_{H}$. Thus, $\mathcal{K}=\bigcup_{H \in \mathcal{K}} \mathcal{K}_{H}$. Then (48) yields the final result (51).

Consider now increasing operators $\Psi$ that are $\mathbb{T}_{a}^{\prime}$-invariant, i.e. invariant to all compositions of horizontal translations $\mu$ and dual vertical translations $\lambda^{\prime}$ as well as invariant to the adjoint $\lambda^{\prime \leftarrow}$ of any dual vertical translation, where $\left(\lambda^{\prime}, \lambda^{\prime \leftarrow}\right)$ is a scalar adjunction. To find kernel representations for such $\Psi$ we need to define the various kernel-related concepts in a dual way. Next we list the basic ideas and results without proof.
(Their derivation can be obtained by using duality of the previous roofs.) The dual kernel of a $\mathbb{T}_{a}^{\prime}$-invariant operator $\Psi$ is defined by

$\operatorname{Ker}^{\prime}(\Psi) \triangleq\left\{F: \Psi(F) \leq q^{\prime}\right\}=\left\{F: \Psi(F)(\overrightarrow{0}) \leq \mathbb{V}_{\mathrm{id}}^{\prime}\right\}$

where $\mathbb{V}_{\text {id }}^{\prime}$ is the identity element of the monoid $\left(\mathbb{V}, \star^{\prime}\right)$.

Example: Let $\mathcal{E}_{H^{\prime}}(F)=F \star^{\prime} H^{\prime}$ be a $\mathbb{T}^{\prime}$-invariant erosion as in (41) and let $\Delta_{H^{\prime}}$ be its adjoint dilation in (42). The dual kernel of this dilation is

$$
\mathcal{K}_{H^{\prime}}^{\prime} \triangleq \operatorname{Ker}^{\prime}\left(\Delta_{H^{\prime}}\right)=\left\{F: F \leq H^{\prime}\right\}
$$

The properties of the dual kernel include the following.

Proposition 4. Consider $\mathbb{T}_{a}^{\prime}$-operators on the signal clodum.

(a) If $\Psi$ is increasing and $F \in \operatorname{Ker}^{\prime}(\Psi)$, then $G \in$ $\operatorname{Ker}^{\prime}(\Psi)$ for all $G \leq F$.

(b) If $\left\{\Psi_{i}: i \in J\right\}$ is an indexed family of operators, then

$$
\operatorname{Ker}^{\prime}(\Psi)=\bigcap_{i} \operatorname{Ker}^{\prime}\left(\Psi_{i}\right) \Rightarrow \Psi=\bigcap_{i} \Psi_{i}
$$

The above results lead us to the following fundamental representation.

Theorem 4. Let $\Psi$ be an increasing $\mathbb{T}_{a}^{\prime}$-invariant operator on the signal clodum $\mathcal{S}$ and let $\left(\mathcal{E}_{H^{\prime}}, \Delta_{H^{\prime}}\right)$ be adjunctions where $\mathcal{E}_{H^{\prime}}(F)=F \star^{\prime} H^{\prime}$ are inf- $\star^{\prime}$ convolutions by functions $H^{\prime}$ in the dual kernel of $\Psi$. Then, $\Psi$ can be represented as the infimum of all the adjoint dilations:

$$
\Psi(F)=\bigwedge_{H^{\prime} \in \operatorname{Ker}^{\prime}(\Psi)} \Delta_{H^{\prime}}(F)
$$

\section{Special Cases}

By specifying $\mathbb{V}$ and the 'multiplication' $\star$ and its dual $\star^{\prime}$, we obtain a large variety of classes of nonlinear image processing systems that are described by the previous unified representations. Next we briefly describe three such choises. 


\subsection{Max-Sum Image Operators}

We set $\mathbb{V}=\overline{\mathbb{R}}=\mathbb{R} \cup\{-\infty,+\infty\}$. The 'multiplications' $\star$ and $\star^{\prime}$ are the regular extended addition + and its dual $+^{\prime}$ over $\overline{\mathbb{R}}$, respectively; i.e., + and $+^{\prime}$ are identical for finite reals, but $a+(-\infty)=-\infty$ and $a+^{\prime}(+\infty)=+\infty$ for all $a \in \overline{\mathbb{R}}$. Thus, the clodum of scalars is $\left([-\infty, \infty], \vee, \wedge,+,+^{\prime}\right)$. In this case $\mathbb{V}$ is a blog and contains an additive group $(\mathbb{R},+)$ where each scalar $a$ has an inverse $-a$ that coincides with its conjugate $a^{*}$. The adjunction $\left(\mathcal{E}_{H}, \Delta_{H}\right)$ of a sup-sum convolution (dilation) $\Delta_{H}$ and its adjoint erosion $\mathcal{E}_{H}$ become

$$
\begin{aligned}
& \Delta_{H}(F)(x)=(F \oplus H)(x) \triangleq \bigvee_{y \in \mathbb{E}} F(y)+H(x-y) \\
& \mathcal{E}_{H}(F)(x)=(F \ominus H)(x) \triangleq \bigwedge_{y \in \mathbb{E}} F(y)-H(y-x)
\end{aligned}
$$

These are the traditional Minkowski dilation and erosion of an image $F$ by an additive structuring function $H$, which have found numerous applications in nonlinear filtering, image processing and computer vision $[9,13,15,24,37,40]$. Similarly, the adjunction $\left(\mathcal{E}_{H^{\prime}}, \Delta_{H^{\prime}}\right)$ of an inf-sum convolution (erosion) $\mathcal{E}_{H^{\prime}}$ and its adjoint dilation $\Delta_{H^{\prime}}$ become

$$
\begin{aligned}
\mathcal{E}_{H^{\prime}}(F)(x) & =\left(F \oplus^{\prime} H\right)(x) \triangleq \bigwedge_{y \in \mathbb{E}} F(y)+{ }^{\prime} H^{\prime}(x-y) \\
\Delta_{H^{\prime}}(F)(x) & =\bigvee_{y \in \mathbb{E}} F(y)+{ }^{\prime}\left[-H^{\prime}(y-x)\right]
\end{aligned}
$$

Note that sup-sum $\oplus$ and inf-sum $\oplus^{\prime}$ convolutions have been known in optimization [2] and convex analysis [34] under the names 'supremal' and 'infimal convolution'.

The signal translations become $\tau_{h, v}(F)(x)=F(x-$ $h)+v$ and their collection $\mathbb{T}$ is a commutative group of automorphisms, studied in [15]. The kernel representations of increasing $\mathbb{T}$-operators as a supremum of Minkowski erosions or as infimum of dilations has been well studied in [21, 24].

In short, the max-sum case is the algebraically richest and most well explored case in mathematical morphology and in minimax algebra both in theory and in applications.

\subsection{Max-Product Image Operators}

Another less explored paradigm, but equally algebraically rich with the max-sum case, results when we choose as set of scalars the extended nonnegative numbers $\mathbb{V}=[0,+\infty]$ and as self-dual $\star$-'multiplication' the standard product ' $x$ ' of nonnegative numbers extended to include the $+\infty$. Thus, the clodum of scalars is $\left([0, \infty], \vee, \wedge, \times, \times^{\prime}\right)$. The signal translations become $\tau_{h, v}(F)(x)=F(x-h) \times v$ and their collection $\mathbb{T}$ is a commutative group of automorphisms, studied in [15]. As in the max-sum case, the scalar set $\mathbb{V}$ is again a blog and contains a multiplicative group $((0, \infty), \cdot)$ with inverses $a^{-1}$ that coincide with the conjugate $a^{*}$ of each scalar $a$. Now, the adjunction $\left(\mathcal{E}_{H}, \Delta_{H}\right)$ of a sup-product convolution (dilation) $\Delta_{H}$ and its adjoint erosion $\mathcal{E}_{H}$ become

$$
\begin{aligned}
\Delta_{H}(F)(x) & =(F \otimes H)(x) \triangleq \bigvee_{y \in \mathbb{E}} F(y) \times H(x-y) \\
\mathcal{E}_{H}(F)(x) & =\bigwedge_{y \in \mathbb{E}} F(y) / H(y-x)
\end{aligned}
$$

These are translation-invariant Minkowski-like dilation and erosion of an image $F$ by a multiplicative structuring function $H$. Some of its properties, their translation-invariances and kernel representations of such systems have been studied in [15].

Note that there is an isomorphism between the maxsum and the max-product enabled by a logarithmicexponential pointwise bijection of the image signals. Despite this isomorphism, we believe that there is a significant applications potential in this algebraic system, which has not been explored so far. This potential can be appreciated by the following remarks: (i) Image signals are naturally nonnegative and the maxproduct dilations-erosions maintain this nonnegativity of the input signals. (ii) In certain vision applications there is sometimes the need to include in the visual processing the logarithm of intensity images; e.g., such a nonlinearity approximates some of the early stages in biological vision systems. This creates the density (log-intensity) representation of images. Then, max-sum dilations-erosions of the density image are equivalent to max-product dilations-erosions of the intensity image.

\section{Lattice Operators Using Fuzzy Norms}

This section presents an important special case of our unified latice image processing systems which 
covers a broad area in the intersection of mathematical morphology and fuzzy logic. We set $\mathbb{V}=[0,1]$ and the clodum of scalars is $\left([0,1], \vee, \wedge, T, T^{\prime}\right)$ where $T\left(T^{\prime}\right)$ is a fuzzy intersection (union). The signal space $\mathcal{S}=\operatorname{Fun}(\mathbb{E},[0,1])$ consists of all image signals defined on $\mathbb{E}=\mathbb{R}^{m}$ or $\mathbb{Z}^{m}$ and assuming real values in $[0,1]$; alternatively, these signals can be viewed as membership functions of fuzzy sets. This is a more difficult clodum case than the previous two (max-sum and max-product) because it is not a blog. Thus, there are no inverses under the $\star$-'multiplication'.

\subsection{Mathematical Morphology and Fuzzy Logic}

Mathematical morphology (MM) and fuzzy sets share many common theoretical concepts. As an earlier example, the use of $\min / \max$ to extend the intersection/union of ordinary (crisp) sets to fuzzy sets [41] has also been used to extend the set-theoretic morphological shrink/expand operations on binary images to $\mathrm{min} / \mathrm{max}$ filtering on graylevel images [10, 31]. While the field of morphological image analysis was maturing, several researchers developed various other approaches using fuzzy logic ideas for extending or generalizing the morphological image operations [4, 39]. The main ingredients of these approaches have been to (1) map the max-plus structure of Minkowski signal dilation to a sup- $T$ signal convolution, where $T$ is some fuzzy intersection norm, and (2) use duality to map the inf-minus structure of Minkowski signal erosion to a inf- $T^{\prime}$ convolution, where $T^{\prime}$ is a dual fuzzy union norm. We refer the reader to [30] for connections and comparisons of all these approaches to fuzzy morphologies. The main disadvantage of these approaches is that composition of the operators from steps (1) and (2) is not guaranteed to be an algebraic opening or closing. In addition to the above approaches, there have been efforts to combine MM and fuzzy logic or lattices and neuro-fuzzy systems by fuzzifying respectively the inclusion indicator or the partial ordering of the lattice, as done respectively in [5] and [17].

In some of our previous work [25, 27, 28] we used lattice theory to develop generalizations of morphological signal and vector operations based on fuzzy norms that have an adjunction structure. In this paper we present these preliminary results as special cases of the general algebraic structure. From fuzzy set theory [19] we use $t$-norms and $t$-conorms to extend intersection and union of crisp sets to signal convolutions. To form openings and closings we use pairs of $t$-norms and fuzzy implications. (A work similar to our lattice-fuzzy theoretical analysis appeared recently in [8].)

\subsection{Fuzzy Intersection and Union Norms}

A fuzzy intersection norm, in short a $T$-norm, is a binary operation $T:[0,1]^{2} \rightarrow[0,1]$ that satisfies the following conditions [19]: For all $a, b, c \in[0,1]$

F1. $T(a, 1)=a$ and $T(a, 0)=0$ (boundary conditions).

F2. $T(a, T(b, c))=T(T(a, b), c)$ (associativity).

F3. $T(a, b)=T(b, a)$ (commutativity).

F4. $b \leq c \Rightarrow T(a, b) \leq T(a, c)$ (increasing). For the $T$-norm to be a scalar dilation (with respect to any argument) on $\mathbb{V}$, it must also satisfy [25]:

F5. $T$ is a continuous function.

A fuzzy union norm [19] is a binary operation $U$ : $[0,1]^{2} \rightarrow[0,1]$ that satisfies F2-F5 and a dual boundary condition:

$\mathrm{F} 1^{\prime} . U(a, 0)=a$ and $U(a, 1)=1$.

Clearly, $U$ is an erosion on $\mathbb{V}$.

\subsection{Lattice Fuzzy Convolutions and Adjunctions}

We have built the general DTI or ETI systems (equivalent to sup- $\star$ or inf- $\star^{\prime}$ convolutions) as sup or inf of signal translations of the type $\tau_{h, v}(f)(x)=f(x-y) \star v$. In this section we shall use new translations where the binary operation $a \star b$ is replaced by fuzzy intersection norms $T$ and the dual operation $a \star^{\prime} b$ is replaced by fuzzy union norms $U$. Namely, the new signal translations on $\mathcal{S}=\operatorname{Fun}(\mathbb{E},[0,1])$ are the operators $\tau$ and the dual translations are the operators $\tau^{\prime}$ :

$$
\begin{aligned}
& \tau_{h, v}(f)(x)=T(f(x-y), v) \\
& \tau_{h, v}^{\prime}(f)(x)=U(f(x-y), v)
\end{aligned}
$$

where $(h, v) \in \mathbb{E} \times[0,1]$ and $f(x)$ is an arbitrary input signal. These translations include both horizontal shifts as well as vertical shifts induced by the fuzzy norms. A signal operator on $\mathcal{S}$ is called translation invariant (resp. dual-translation invariant) iff it commutes with any such translation $\tau$ (resp. $\tau^{\prime}$ ) based on a fuzzy norm. 
Consider now the two elementary signals, the impulse $q$ and the dual impulse $q^{\prime}$ :

$$
q(x) \triangleq\left\{\begin{array}{ll}
1, & x=\overrightarrow{0} \\
0, & x \neq \overrightarrow{0}
\end{array}, \quad q^{\prime}(x) \triangleq \begin{cases}0, & x=\overrightarrow{0} \\
1, & x \neq \overrightarrow{0}\end{cases}\right.
$$

Then every signal $f$ can be represented as a supremum of translated impulses or as infimum of dual-translated dual impulses:

$$
\begin{aligned}
f(x) & =\bigvee_{y} T[q(x-y), f(y)] \\
& =\bigwedge_{y} U\left[q^{\prime}(x-y), f(y)\right]
\end{aligned}
$$

Translation invariant signal dilations and erosions can result, respectively, from the sup- $T$ convolution $\bigcirc_{T}$ and the inf- $U$ convolution $\bigcirc_{U}^{\prime}$ of two signals $f$ and $g$ defined by

$$
\begin{aligned}
& (f \bigcirc T g)(x) \triangleq \bigvee_{y} T[g(x-y), f(y)], \\
& \left(f \bigcirc_{U}^{\prime} g\right)(x) \triangleq \bigwedge_{y} U[g(x-y), f(y)]
\end{aligned}
$$

The following results are a direct corollary of our convolution representation Theorem 2 .

Theorem 5. (a) Given a fuzzy intersection norm $T$, an operator $\Delta$ on the signal clodum $\operatorname{Fun}(\mathbb{E},[0,1])$ is a dilation invariant to the general translations (59) iff it can be represented as the sup- $T$ convolution of the input signal with the operator's impulse response $H=\Delta(q)$.

(b) Given a fuzzy union norm $U$, an operator $\mathcal{E}$ on the signal clodum $\mathrm{Fun}(\mathbb{E},[0,1])$ is an erosion invariant to the dual translations (60) iff it can be represented as the inf-U convolution of the input signal with the system's dual impulse response $H^{\prime}=\mathcal{E}\left(q^{\prime}\right)$.

However, the erosion $\mathcal{E}$ of the above theorem may $n o t$ be the adjoint of the dilation $\Delta$. To form an adjunction, we first define a signal fuzzy dilation as a sup- $T$ convolution:

$$
\begin{aligned}
\Delta_{H, T}(F)(x) & \triangleq \bigvee_{y \in \mathbb{E}} T[H(x-y), F(y)] \\
& =\left(F \bigcirc_{T} H\right)(x)
\end{aligned}
$$

By recognizing $T[H(x-y), F(y)]$ as the scalar dilations $d_{y, x}(F(y))$ in the general decomposition (19) of a signal dilation, it follows that the adjoint signal fuzzy erosion is

$$
\mathcal{E}_{H, \Omega}(G)(y) \triangleq \bigwedge_{x \in \mathbb{E}} \Omega[H(x-y), G(x)]
$$

where $\Omega[H(x-y), G(x)]$ represents the adjoint scalar erosions $e_{x, y}(G(x))$ in (19) and is actually the adjoint of the fuzzy $T$-norm:

$$
T(a, v) \leq w \Leftrightarrow v \leq \Omega(a, w)
$$

An alternative interpretation of $T[H(x-y), F(y)]$ and $\Omega[H(x-y), G(x)]$ is that they are equal, respectively, to a scalar V-translation $\lambda$ and its adjoint $\lambda^{\leftarrow}$ of the signal values:

$$
\begin{aligned}
T[H(x-y), F(y)] & =\lambda_{H(x-y)}(F(y)), \\
\Omega[H(x-y), G(x)] & =\lambda_{H(x-y)}^{\leftarrow}(G(x))
\end{aligned}
$$

Now, given $T$ we can find its adjoint function $\Omega$ by

$$
\Omega(a, w)=\sup \{v \in[0,1]: T(a, v) \leq w\}
$$

In fuzzy logic, the norm $T$ can be interpreted as a logical conjunction, whereas its corresponding adjoint $\Omega$ can be interpreted as a logical implication [19].

Three examples of $T$-norms are:

$$
\begin{aligned}
& \text { Min : } T_{1}(a, v)=\min (a, v) \\
& \text { Product : } T_{2}(a, v)=a \cdot v \\
& \text { Yager : } T_{3}(a, v)=1-\left(1 \wedge \left[(1-v)^{p}\right.\right. \\
& \left.\left.\quad+(1-a)^{p}\right]^{1 / p}\right), p>0 .
\end{aligned}
$$

The corresponding three adjoint functions are:

$$
\begin{aligned}
& \Omega_{1}(a, w)= \begin{cases}w, & w<a \\
1, & w \geq a\end{cases} \\
& \Omega_{2}(a, w)= \begin{cases}\min (w / a, 1), & a>0 \\
1, & a=0\end{cases} \\
& \Omega_{3}(a, w)=\left\{\begin{array}{l}
1-\left[(1-w)^{p}-(1-a)^{p}\right]^{1 / p}, \\
w<a \\
1, \quad w \geq a
\end{array}\right.
\end{aligned}
$$

Let us consider now the construction of lattice-fuzzy openings and closings based on an adjunction $(\varepsilon, \delta)$ of 


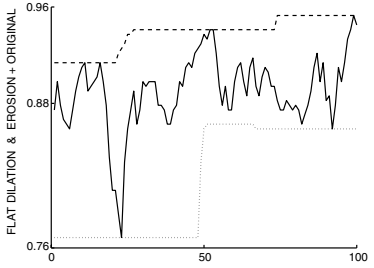

(a)

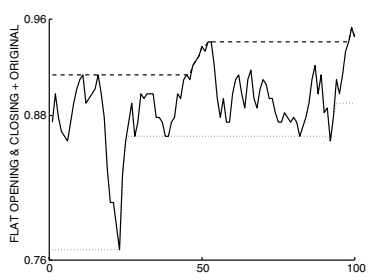

(e)

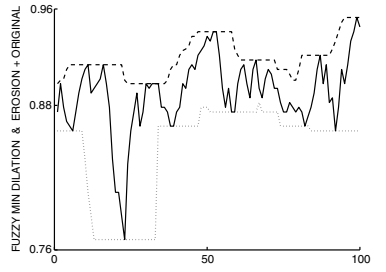

(b)

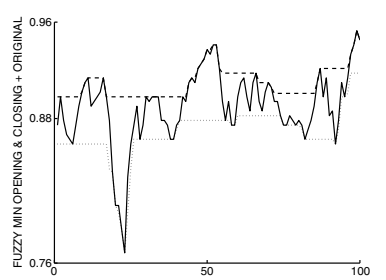

(f)

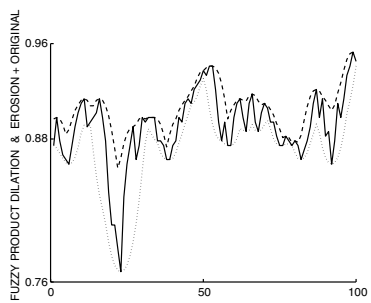

(c)

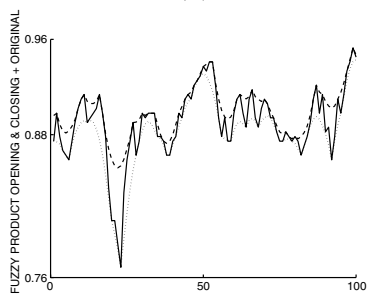

(g)

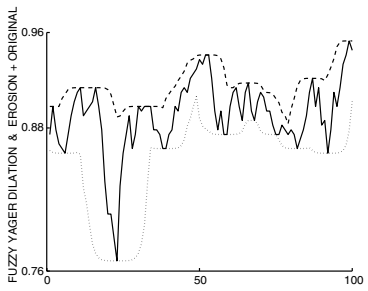

(d)

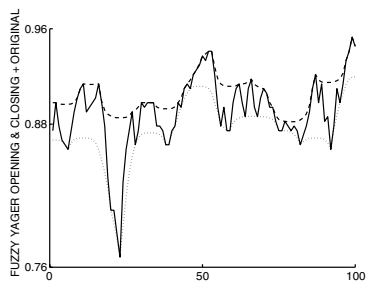

(h)

Figure 1. Comparison of 1D basic morphological and lattice-fuzzy signal operators. Rows 1 and 2, left to right: flat, minimum, product, Yager. Row 1: original signal (solid line), dilation (dashed line), erosion (dotted line). Row 2: closing (dashed line), opening (dotted line). Courtesy of $[27]$.

a lattice-fuzzy dilation $\delta$ and erosion $\varepsilon$. The adjunctional lattice-fuzzy opening $\alpha$ and lattice-fuzzy closing $\beta$ are defined as

$$
\alpha(f) \triangleq \delta(\varepsilon(f)), \quad \beta(f) \triangleq \varepsilon(\delta(f))
$$

This is the correct approach to create openings and closings from fuzzy dilations and erosions. To compare it with previous works, consider an involutive fuzzy complement $a \mapsto a^{*}$, e.g. $a^{*}=1-a$. This is a negation (i.e. conjugation) on the scalar clodum $[0,1]$ and induces a negation on the signal clodum $\mathcal{S}$ too. If we define via complementation an alternative erosion operator (as an inf- $U$ convolution) by

$$
\varepsilon^{\prime}(f)(y)=\bigwedge_{x} U[f(x), h(y-x)]
$$

where $U(a, b)=1-T(1-a, 1-b)$ is a fuzzy union that is the dual (i.e. complement) of the fuzzy intersection $T$, then $\varepsilon^{\prime}(f)=1-\delta(1-f)=\delta^{*}(f)$, where $\psi^{*}$ denotes the negative operator of $\psi$; i.e., this second erosion $\varepsilon^{\prime}$ is the dual (i.e. negative) of the first dilation $\delta$. Further, the adjoint dilation $\delta^{\prime}$ of $\varepsilon^{\prime}$ is an operator that is dual (i.e. negative) of the first erosion $\varepsilon$. Many previous works used pairs $\left(\varepsilon^{\prime}, \delta\right)$ which are duality pairs (via negation) but not adjunctions and hence cannot form openings/closings via compositions.

\subsection{Experiments}

To gain some insight on the lattice-fuzzy image operators, we briefly present a few experimental results illustrating the differences between the classical morphological operators and the lattice operators based on fuzzy $T$-norms.

Figure 1 reports experiments with 1D images and shows the outputs of dilation, erosion, opening and closing operators, first for the morphological type using a 51-pixel flat structuring element and second for the fuzzy type (62), (63) and (67). For the fuzzy operations in Fig. 1, we used three $T$-norms (the minimum norm, the product norm and the Yager norm with parameter $p=2$ ) and a parabolic non-flat structuring function $H: \mathbb{Z} \rightarrow[0,1]$

$$
H(x)= \begin{cases}1-k(x / s)^{2}, & |x| \leq s \\ 0, & |x|>s\end{cases}
$$

The parameter $s$ determines the scale, while $k$ affects the shape of $H$. (We used $s=25$ and $k=0.5$.) In general, by experimenting with a large variety of $T$-norms and structuring functions $H$ we have observed that, the fuzzy operators are more adaptive and track closer the peaks/valleys of the signal than the corresponding flat morphological operators of the same scale.

Figure 2 reports experiments with 2D images. For all the fuzzy operations in this figure, we used the Yager 


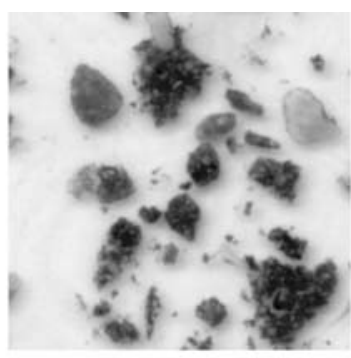

(a)

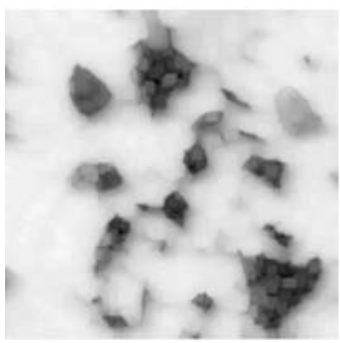

(b)

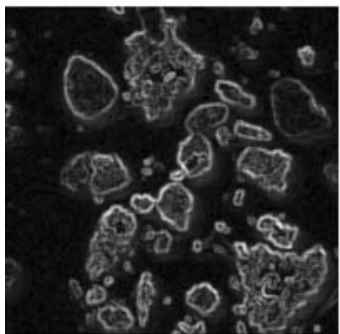

(f)

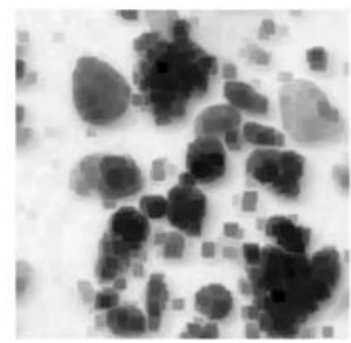

(c)

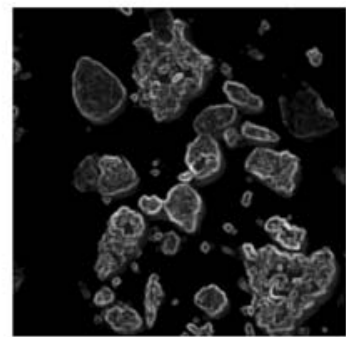

(g)

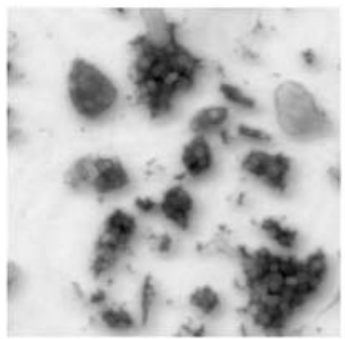

(d)

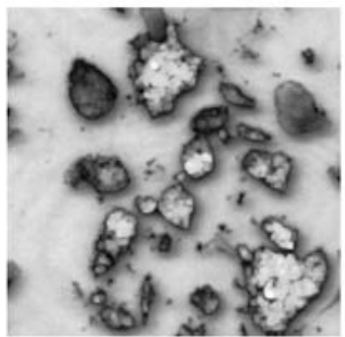

(h)

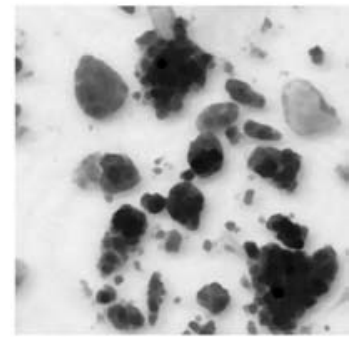

(e)

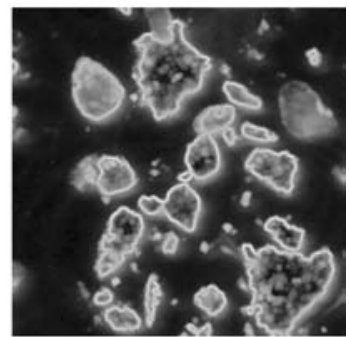

(i)

Figure 2. (a) Original image $F$. (b) Morphological flat dilation $F \oplus B$. (c) Morphological flat erosion $F \ominus B$. (d) Fuzzy dilation $\delta(F)$. (c) Fuzzy erosion $\varepsilon(F)$. (f) Morphological gradient $F \oplus B-F \ominus B$. (g) $\delta(F)-\varepsilon(F)$. (h) Fuzzy min gradient $\min [\delta(F), 1-\varepsilon(F)]$. (i) Fuzzy max gradient $\max [\delta(F), 1-\varepsilon(F)]$. Courtesy of [27].

$T$-norm with parameter $p=10$ and a conical structuring function $H$. The second row compares the morphological flat dilation and erosion of an original image in Fig. 2(a) with its fuzzy dilation and erosion. In both cases the structuring element had a $7 \times 7$-pixel support, being flat in the morphological case and conical in the fuzzy case. The third row of Fig. 2 deals with edge enhancement: Figure 2(f) shows the standard discrete morphological gradient $F \oplus B-F \ominus B$, as the difference between the morphological flat dilation and erosion, respectively, of $F$ by a $3 \times 3$-pixel square $B$. Figure $2(\mathrm{~g})$ shows the same type of gradient but uses a fuzzy dilation $\delta$ and erosion $\varepsilon$ with a $3 \times 3$-pixel structuring function $H$. Figures $2(\mathrm{~h}$ ) and (i) combine the fuzzy dilation and erosion differently to derive respectively the following two types of new edge gradients:

$$
\begin{aligned}
& \text { FuzzyEdge }_{\text {min }}(F)=\min [\delta(F), 1-\varepsilon(F)] \\
& \text { FuzzyEdge }_{\text {max }}(F)=\max [\delta(F), 1-\varepsilon(F)]
\end{aligned}
$$

The new edge gradients were inspired by the standard discrete morphological gradient $F \oplus B-F \ominus B$, but to make the gradient operator more consistent with fuzzy set theory we replaced the difference between dilation and erosion with min (or max) of the dilation and the fuzzy complement of the erosion. As shown in Fig. 2, these new fuzzy gradient operators have a quite promising behavior since they yield cleaner and sharper edge peaks than the morphological gradient. 
The power but also the difficulty in applying these lattice fuzzy operators to image analysis is the large variety of fuzzy norms and the absence of systematic ways in selecting them. Towards this goal, we have experimented in $[27,28]$ with applying these fuzzy operators to various nonlinear filtering and image analysis tasks, attempting to understand the effect that the type of fuzzy norm and the shape-size of structuring function have on the resulting new image operators. This preliminary work showed that, by experimenting with the type of fuzzy norm and the shape-size of the structuring function, it is possible to adapt the new fuzzy operators so that their performance has many promising aspects compared with the standard morphological operators. For example, in the problem of edge detection, it is possible to optimize the shape and size of the structuring function so that the edge derivatives have sharper peaks [27]. Further, the edge gradients based on lattice fuzzy operators have shown improved performance in noise [28]. Thus, by combining lattice-based MM and fuzzy set theory, we can create new operators, like the fuzzy edge gradients, that extend and improve the capabilities of the standard morphological operators.

\section{Conclusions}

We have obtained several results that extend the algebraic theory of mathematical morphology in three directions: (1) Development of a general algebraic structure for signals and images that is minimally sufficient for both translation-invariant morphology (max-sum and max-product convolutions) as well as for minimax signal algebra and nonlinear image processing based on fuzzy logic. (2) Unification of convolutional representations of translation-invariant signal operators that obey max $-\star$ superpositions via nonlinear sup- $\star$ convolutions over a clodum, i.e. a signal space which combines the sup-inf lattice structure with a scalar semiring arithmetic that possesses generalized 'additions' and $\star$-‘multiplications'. (3) Unification of kernel representations of increasing and translation-invariant signal operators over a clodum as suprema of erosion-type (or infima of dilation-type) nonlinear convolutions.

Our results provide some theoretical support and open the way in morphological image processing for future applications that are based (i) on minimax signal algebra (e.g. minimax signal matrix and eigenvalueeigenvector analysis) and (ii) on lattice fuzzy image operators that are more adaptive than classic morphological operators, are amenable to optimal designs, and are closely related to popular pattern recognition systems of the neuro-fuzzy network type. We also note that the lattice fuzzy case includes as special case the Boolean image algebra and hence all the morphological operators used in binary image processing. Finally we note that our results provide a bridge $[25,26]$ between morphological image and signal processing and discrete event control systems based on minimax algebra, both for the max-sum and the max-fuzzy cases. This can cause a cross-fertilization of the two fields.

\section{Acknowledgments}

This research work was supported by the Greek General Secretariat for Research and Technology Union under the program חENE $\Delta-2001$ with Grant \# 01E $\Delta 431$ and by the European Union under the Network of Excellence MUSCLE. The author wishes to thank his collaborators V. Tzouvaras and G. Stamou at NTUA for insightful discussions and experiments on lattice fuzzy image operators.

\section{Notes}

1. If in the semilattice $(\mathcal{P}, \circ)$ we consider a different partial ordering $X \leq^{\prime} Y \leftrightarrow Y=X \circ Y$ where $X \circ Y$ is interpreted as the infimum $X \wedge Y$, then $(\mathcal{P}, \circ)$ becomes an inf-semilattice and vice-versa. Obviously, $\left(\mathcal{P}, \leq^{\prime}\right)$ is the dual poset of $(\mathcal{P}, \leq)$.

2. In minimax algebra [7], a semilattice is called a band. Further, a semilattice-ordered semigroup is called a belt, and a latticeordered double semigroup is called a belt with duality. A belt $(\mathcal{B}, \vee, \star)$ with an identity element for the semigroup operation $\star$ and with an element $\zeta$ that is both the least element w.r.t. $\leq$ and also a null, i.e. $a \vee \zeta=a$ and $a \star \zeta=\zeta, \forall a \in \mathcal{B}$, is called a dioid in [6].

\section{References}

1. G.J.F. Banon and J. Barrera, "Minimal representations for translation-invariant set mappings by mathematical Morphology", SIAM J. Appl. Math., Vol. 51, pp. 1782-1798, 1991.

2. R. Bellman and W. Karush, "On the maximum transform", $J$. Math. Anal. Appl., vol. 6, pp. 67-74, 1963.

3. G. Birkhoff, Lattice Theory, Amer. Math. Soc.: Providence, Rhode Island, 1967.

4. I. Bloch and H. Maitre, "Fuzzy mathematical morphologies: A comparative study," Pattern Recognition 28, Vol. 9, pp. 13411387, 1995.

5. V. Chatzis and I. Pitas, "A generalized fuzzy mathematical morphology and its application in robust 2-D and 3-D object representation," IEEE Trans. Image Process., Vol. 9, pp. 1798-1810, 2000.

6. G. Cohen, P. Moller, J.P. Quadrat, and M. Viot, "Algebraic tools for the performance evaluation of discrete event systems," Proc. IEEE, Vol. 77, pp. 39-58, 1989. 
7. R. Cuninghame-Green, Minimax Algebra, Springer-Verlag: New York, 1979.

8. T.Q. Deng and H.J.A.M. Heijmans, "Grey-scale morphology based on fuzzy logic," J. Math. Imaging and Vision, 16, 155171, 2002.

9. E.R. Dougherty and J. Astola, An Introduction to Nonlinear Image Processing, SPIE Press: Bellingham, Washington, Vol. TT16, 1994.

10. V. Goetcherian, "From binary to greytone image processing using fuzzy logic concepts," Pattern Recognition, Vol. 12, pp. 7-15, 1980.

11. J. Goutsias, "Morphological analysis of discrete random shapes," J. Math. Imaging and Vision, Vol. 2, 193-215, 1992.

12. F. Guichard and J.-M. Morel, Image Analysis and PDEs, Book to appear.

13. R.M. Haralick and L.G. Shapiro, Computer and Robot Vision, Vol. I, Addison-Wesley, 1992.

14. H.J.A.M. Heijmans, "Mathematical Morphology: An algebraic approach," CWI Newsletter, Vol. 14, 7-27, 1987.

15. H.J.A.M. Heijmans, Morphological Image Operators, Acad. Press: Boston, 1994.

16. H.J.A.M. Heijmans and C. Ronse, "The algebraic basis of mathematical morphology. Part I: Dilations and erosions," Computer Vision, Graphics, and Image Processing, Vol. 50, pp. 245-295, 1990.

17. V.G. Kaburlasos and V. Petridis, "Fuzzy lattice neurocomputing (FLN) models," Neural Networks, Vol. 13, pp. 1145-1169, 2000

18. R. Keshet (Kresch), "Mathematical morphology on complete semilattices and its applications to image processing," Fundamentae Informatica, Vol. 41, pp. 33-56, 2000.

19. G.J. Klir and B. Yuan, Fuzzy Sets and Fuzzy Logic: Theory and Applications, Prentice-Hall, 1995.

20. R.P. Loce and E.R. Dougherty, "Optimal morphological restoration: The morphological filter mean-absolute-error theorem," $J$. Visual Communication \& Image Representation, Vol. 3, No. 4, pp. 412-432, 1992.

21. P. Maragos, "A representation theory for morphological image and signal processing," IEEE Trans. Pattern Analysis and Machine Intelligence, Vol. 11, pp. 586-599, 1989.

22. P. Maragos, "Morphological systems: Slope transforms and max-min difference and differential equations," Signal Processing, Vol. 38, pp. 57-77, 1994.

23. P. Maragos, "Morphological signal and image processing," in The Digital Signal Processing Handbook, V. Madisetti and D. Williams (Ed.), CRC and IEEE Press, 1998.

24. P. Maragos and R.W. Schafer, "Morphological systems for multidimensional signal processing," Proc. IEEE, Vol. 78, pp. 690710,1990

25. P. Maragos, G. Stamou, and S. Tzafestas, "A lattice control model of fuzzy dynamical systems in state-space", in Mathematical Morphology and Its Application to Image and Signal Processing, J. Goutsias, L. Vincent and D. Bloomberg (Eds.), Kluwer Acad. Publ.: Boston, 2000, pp. 61-70.

26. P. Maragos and S. Tzafestas, "Max-Min Control Systems with Applications to Discrete Event Dynamical Systems," in Advances in Manufacturing: Decision, Control and Information Technology, S.G. Tzafestas (Ed.), Springer-Verlag, 1999, pp. $217-230$

27. P. Maragos, V. Tzouvaras, and G. Stamou, "Synthesis and applications of lattice image operators based on fuzzy norms", in
Proc. Int'l Conf. Image Processing (ICIP-2001), Thessaloniki, Greece, Oct. 2001.

28. P. Maragos, V. Tzouvaras, and G. Stamou, "Lattice fuzzy image operators and generalized image gradients", in Proc. Int'l Fuzzy Systems Assoc. World Congress (IFSA-2003), Turkey, July 2003. LNCS 2715, Springer-Verlag, 2003, pp. 412-419.

29. G. Matheron, Random Sets and Integral Geometry, J. Wiley: NY, 1975.

30. M. Nachtegael and E.E. Kerre, "Connections between binary, gray-scale and fuzzy mathematical morphologies," Fyzzy Sets and Systems, Vol. 124, pp. 73-85, 2001.

31. Y. Nakagawa and A. Rosenfeld, "A note on the use of local min and max operations in digital picture processing," IEEE Trans. Syst., Man, Cybern., Vol. SMC-8, pp. 632-635, 1978.

32. G.X. Ritter and P.D. Gader, "Image algebra techniques for parallel image processing," J. Paral. Distr. Comput., Vol. 4, pp. 7-44, 1987.

33. G.X. Ritter and J.N. Wilson, "Image algebra in a nutshell," in Proc. 1st ICCV, London, June 1987, pp. 641-645.

34. R.T. Rockafellar, Convex Analysis, Princeton Univ. Press: Princeton, 1972.

35. J.B.T.M Roerdink, "Mathematical morphology with noncommutative symmetry groups," in Mathematical Morphology in Image Processing, E.R. Dougherty (Ed.), Marcel Dekker: NY, 1993, pp. 205-254.

36. C. Ronse and H.J.A.M. Heijmans, "The algebraic basis of mathematical morphology. Part II: Openings and closings," CVGIP: Image Understanding, Vol. 54, pp. 74-97, 1991.

37. J. Serra, Image Analysis and Mathematical Morphology, Acad. Press: NY, 1982.

38. J. Serra (Ed.), Image Analysis and Mathematical Morphology, Vol. 2: Theoretical Advances, Acad. Press: NY 1988.

39. D. Sinha and E.R. Dougherty, "Fuzzy mathematical morphology," J. Visual Communication and Image Representation, Vol. 3, No. 3, pp. 286-302, 1992.

40. S.R. Sternberg, "Grayscale morphology," Comput. Vision, Graph., Image Proc. Vol. 35, pp. 333-355, 1986.

41. L.A. Zadeh, "Fuzzy sets," Information and Control, Vol. 8, pp. 338-353, 1965.

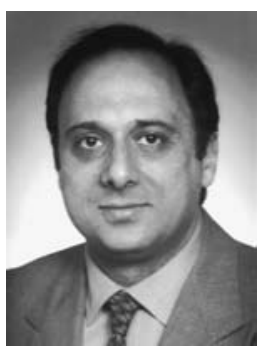

Petros Maragos received the Diploma degree in electrical engineering from the National Technical University of Athens in 1980, and the M.Sc.E.E. and Ph.D. degrees from Georgia Tech, Atlanta, USA, in 1982 and 1985.

In 1985 he joined the faculty of the Division of Applied Sciences at Harvard University, Cambridge, Massachusetts, where he worked for 
8 years as professor of electrical engineering, affiliated with the interdisciplinary Harvard Robotics Lab. He has also been a consultant to several industry research groups including Xerox's research on document image analysis. In 1993, he joined the faculty of the School of Electrical and Computer Engineering at Georgia Tech. During parts of 1996-98 he was on academic leave working as a senior researcher at the Institute for Language and Speech Processing in Athens. In 1998, he joined the faculty of the National Technical University of Athens where he is currently working as professor of electrical and computer engineering. His current research and teaching interests include the general areas of signal processing, systems theory, pattern recognition, and their applications to image processing and computer vision, and computer speech processing and recognition.

He has served as associate editor for the IEEE Trans. on Acoustics, Speech, and Signal Processing, editorial board member for the Journal of Visual Communications and Image Representation, and guest editor for the IEEE Trans. on Image Processing; general chairman for the 1992 SPIE Conference on Visual Communications and Image Processing, and co-chairman for the 1996 International Symposium on Mathematical Morphology; member of two IEEE DSP committees; and president of the International Society for Mathematical Morphology.

Dr. Maragos' research work has received several awards, including: a 1987 US National Science Foundation Presidential Young Investigator Award; the 1988 IEEE Signal Processing Society's Paper Award for the paper 'Morphological Filters'; the 1994 IEEE Signal Processing Society's Senior Award and the 1995 IEEE Baker Award for the paper 'Energy Separation in Signal Modulations with Application to Speech Analysis'; and the 1996 Pattern Recognition Society's Honorable Mention Award for the paper 'Min-Max Classifiers'. In 1995, he was elected Fellow of IEEE for his contributions to the theory and applications of nonlinear signal processing systems. 